\title{
Exercise-induced peptide TAG-23 protects cardiomyocytes from reperfusion injury through regulating PKG-cCbl interaction
}

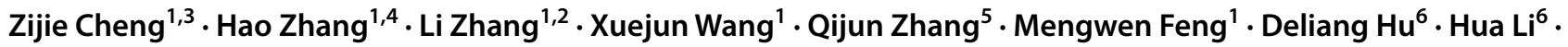 \\ Lingmei Qian ${ }^{1,2}$ (D)
}

Received: 9 November 2020 / Accepted: 11 May 2021 / Published online: 25 June 2021

(c) The Author(s) 2021

\begin{abstract}
Recent studies have revealed that proper exercise can reduce the risk of chronic disease and is beneficial to the body. Peptides have been shown to play an important role in various pathological processes, including cardiovascular diseases. However, little is known about the role of exercise-induced peptides in cardiovascular disease. We aimed to explore the function and mechanism of TAG-23 peptide in reperfusion injury and oxidative stress. Treatment with TAG-23 peptide significantly improved cell viability, the mitochondrial membrane potential, and ROS levels and reduced LDH release, the apoptosis rate and caspase 3 activation in vitro. In vivo, TAG-23 ameliorated MI and heart failure induced by I/R or DOX treatment. Pulldown assays showed that TAG-23 can bind to PKG. The TAG-23-PKG complex inhibited PKG degradation through the UPS. We also identified $\mathrm{cCbl}$ as the E3 ligase of PKG and found that the interaction between these proteins was impaired by TAG-23 treatment. In addition, we provided evidence that TAG-23 mediated Lys48-linked polyubiquitination and subsequent proteasomal degradation. Our results reveal that a novel exercise-induced peptide, TAG-23, can inhibit PKG degradation by serving as a competitive binding peptide to attenuate the formation of the PKG-cCbl complex. Treatment with TAG-23 may be a new therapeutic approach for reperfusion injury.
\end{abstract}

Keywords TAG-23 $\cdot \mathrm{PKG} \cdot \mathrm{cCbl} \cdot$ Doxorubicin $\cdot$ Myocardial infarction

Zijie Cheng, Hao Zhang, Li Zhang, and Xuejun Wang contributed equally to this work.

The corresponding authors have the following order: 1st corresponding author: Lingmei Qian, 2nd corresponding author: Zijie Cheng.

\section{Zijie Cheng}

zjcheng@njmu.edu.cn

$\triangle$ Lingmei Qian

lmqian@njmu.edu.cn

1 Department of Cardiology, The First Affiliated Hospital of Nanjing Medical University, NO.300 Guangzhou Road, Gulou District, Nanjing 210029, China

2 Department of Cardiology, Tongren Hospital Affiliated To Medical School of Shanghai Jiao Tong University, Shanghai, China

3 Department of Cardiology, Zhongshan Hospital, Fudan University, Shanghai, China

4 Department of Internal Medicine, The Affiliated Drum Tower Hospital of Nanjing University Medical School, Nanjing, China

5 Department of Cardiology, The Affiliated People's Hospital of Ningbo University, Ningbo, China

6 Department of Emergency, The First Affiliated Hospital of Nanjing Medical University, Nanjing, China

$\begin{array}{ll}\text { Abbreviations } \\ \text { LDH } & \text { Lactate dehydrogenase } \\ \text { ROS } & \text { Reactive oxygen species } \\ \text { DOX } & \text { Doxorubicin } \\ \text { PKG } & \text { Cyclic GMP-dependent protein kinase } \\ \text { NO } & \text { Nitric oxide } \\ \text { CHX } & \text { Cycloheximide } \\ \text { ERK } & \text { Extracellular signal-related kinases } \\ \text { JNK } & \text { Jun amino-terminal kinases } \\ \text { UPS } & \text { Ubiquitin proteasome system } \\ \text { LVEDd } & \text { Left ventricular end-diastolic dimensions } \\ \text { LVPWd } & \text { Left ventricular posterior wall dimensions } \\ \text { LVPWs } & \text { Left ventricular posterior wall at end systole } \\ \text { EF } & \text { Ejection fraction } \\ \text { FS } & \text { Fractional shortening } \\ \text { LVEDs } & \text { Left ventricular end-diastolic stiffness } \\ \text { PBS } & \text { Phosphate-buffered saline }\end{array}$




\section{Introduction}

Myocardial infarction (MI) is the leading cause of death worldwide [8,33]. Emerging evidence have demonstrated that apoptosis, the inflammatory response, and reactive oxygen species (ROS) are associated with cellular damage during the ischemic period [1]. Cardiomyocyte death resulting from reperfusion injury can be prevented at the end of the ischemic time, and targeting regulated cell death pathways before reperfusion injury appears to be a promising approach to minimize MI injury [4].

It is well established that exercise not only reduces the risks of chronic disease but also provides protection against cardiovascular disease [5]. Exercise is recommended for patients with heart failure or following MI [34]. Recent studies have shown that the mechanism underlying exercise-induced cardioprotection mainly contributes to increasing antioxidant capacity, anti-inflammatory effects, and the secretion of beneficial hormones or cytokines [16, 23]. Exercise-induced bioactive factors, including peptides, hormones, and nucleic acids, are secreted into the circulatory system and allow cell communication within different organs $[12,15]$. These bioactive components may be beneficial for physiological function. However, the underlying mechanisms of exercise-induced cardioprotection remain unknown.

Accumulating evidence has shown that peptides play important roles in various pathophysiological processes. For example, $\mathrm{B} \beta_{15-42}$ can ameliorate leukocyte transmigration across the endothelial monolayer to ameliorate ischemia-reperfusion (I/R) injury [24]. Oral administration of the peptide Val-Pro-Pro, which is derived from casein, reduces proinflammatory macrophage accumulation in adipose tissue [1]. Pretreatment with GLP-1 peptide protects against ischemic cardiac dysfunction and improves heart function during reperfusion [21]. These studies emphasize the importance of peptides in pathological processes. Despite advances in the knowledge of cardiovascular diseases, further identification of bioactive peptides will facilitate better understanding of the mechanism of I/R injury.

Previous studies have quantified peptides that are differentially induced by exercise by 2D-LC-MS/MS, and 5548 unique peptides that are induced at different time points have been screened [23]. In the peptidome, the endogenous peptide TAG-23 attracted our attention because it is upregulated in the plasma during exercise. Live-cell microscopy was utilized to observe the effect of TAG-23 on migration. A wound healing assay showed that compared with a scramble peptide, TAG-23 peptide can increase cell migration by affecting the cell cycle and extracellular matrix remodeling [23]. TAG-23 peptide is derived from transgelin, which is primarily expressed in smooth muscle cells. Transgelin knockout mice show decreased actin content [39]. Transgelin, which is an androgen receptor (AR) inhibitor, can suppress AR function in LNCaP cells by binding with p53 [41]. To date, there have been no reports regarding the role of TAG-23 peptide in cardiovascular disease.

In this study, we aimed to evaluate the function and mechanism of the exercise-induced peptide TAG-23 in reperfusion and oxidative stress. Our study demonstrated that TAG23 confers cardioprotective effects against MI and serves as a competitive peptide to attenuate the PKG-cCbl interaction. We also provide evidence that TAG-23 mediates PKG degradation at the Lys 48 site. Taken together, these results are the first to demonstrate the function and mechanism of exerciseinduced peptides in cardiovascular disease and provide a new clue for understanding MI.

\section{Materials and methods}

\section{Cell culture}

Rat primary cardiomyocytes were extracted one day after birth. The ventricles were harvested, rinsed briefly in $75 \%$ ethanol and maintained in DMEM. After the heart tissues were cut into small fragments, they were washed twice with PBS and then transferred to digestion solution (0.4\% type 2 collagenase $/ 0.6 \%$ pancreatin). Repeat digestion was performed at $37^{\circ} \mathrm{C}$ for $20 \mathrm{~min}$. The supernatant was collected after every round of digestion, and the cells were centrifuged at $1000 \mathrm{rpm}$ for $5 \mathrm{~min}$. Cardiac fibroblasts were minimized by pre-plating the cells for $2 \mathrm{~h}$. Cardiomyocytes in the suspension were seeded on gelatin-coated culture plates (G1393, Sigma-Aldrich, USA). The cells were then cultured on gelatinized plates for $24-48 \mathrm{~h}$ before further study.

To induce hypoxia/reperfusion (H/R), injury cells were treated with $\mathrm{H} / \mathrm{R}$ buffer in a hypoxic chamber (BillupsRothenberg), and the chamber was flushed with $95 \% \mathrm{~N}_{2} / 5 \%$ $\mathrm{CO}_{2}$ for $30 \mathrm{~min}$. Then, the chamber was closed for an additional $4 \mathrm{~h}$. The cells were washed twice with PBS, and the culture medium was replenished. Neonatal rat ventricular myocytes (NRVMs) incubated in normoxic medium (in $\mathrm{mM}$ : 4 HEPES, $137 \mathrm{NaCl}, 3.8 \mathrm{KCl}, 0.9 \mathrm{CaCl}_{2}, 0.49 \mathrm{MgCl}_{2}$, and 5.6 D-glucose, $\mathrm{pH}$ 7.4) were used as controls.

The sequence of TAG-23 was MGSNRGASQAGMTGYGRPRQIIS (TAG-23). The scramble peptide was RGMINGRMIQSTGSYPSARGQAG (control). The peptides were synthesized by Scientific Peptide Biological Technology Co., Ltd. (Shanghai, China). The concentration used is indicated in the figure legends. The peptides were added to the culture medium for $1 \mathrm{~h}$ before $\mathrm{H} / \mathrm{R}$ treatment. 


\section{JC-1 assay}

The mitochondrial membrane potential was measured using a mitochondrial membrane potential assay kit with JC-1 according to the manufacturer's instructions. The NRVMs were cultured in serum-free DMEM containing $(1 \times)$ JC- 1 staining working solution at $37{ }^{\circ} \mathrm{C}$ for $20 \mathrm{~min}$. Then, the cells were washed twice with JC-1 buffer, $2 \mathrm{ml}$ of DMEM was added, and the cells were photographed with a fluorescence microscope (BX61; Olympus Corporation, Tokyo, Japan).

\section{ROS levels}

The levels of intracellular ROS were determined using a ROS assay kit (Beyotime, Shanghai, China) according to the instructions. NRVMs were incubated in serum-free DMEM containing $0.1 \%$ 2', 7' -dichlorofluorescein diacetate (DCFHDA) at $37^{\circ} \mathrm{C}$ for $20 \mathrm{~min}$, washed three times with serum-free DMEM and photographed with a fluorescence microscope.

\section{TUNEL staining}

NRVMs were seeded in 6-well plates $\left(1 \times 10^{5}\right.$ cells per well). After H/R treatment, the cells were washed twice with PBS and fixed with $4 \%$ paraformaldehyde. Apoptotic cells were visualized by TUNEL staining according to the manufacturer's protocol (Promega). The TUNEL fluorescence intensity/DAPI fluorescence intensity was used to calculate the percentage of positively stained cells, and the density was evaluated using Image J software 1.26 (Wayne Rasband, National Institutes of Health, Bethesda, MD, USA).

\section{Cell viability}

NRVMs were seeded on 96-well plates (1500 cells/well). After $24 \mathrm{~h}$, the cells were treated with different concentrations of TAG-23, and cell viability was measured by the CCK8 assay using a cell viability assay kit (Promega) according to the manufacturer's protocols.

\section{MI}

I/R injury was induced in wild-type male mice (8 weeks) on a C57/B6 background. Mice were sedated with 5\% isoflurane- $\mathrm{O}_{2}$ (cat.no. 792632; Sigma-Aldrich; Merck KGaA) balanced mixture (maintained at $1.5 \%$ ). All mice were anaesthetized by breathing a 5\% isoflurane- $\mathrm{O}_{2}$ balanced mixture (maintained at $1.5 \%$ ). Mice were confirmed to be deeply anesthetized after they were immobile for $1 \mathrm{~min}$. Left anterior descending occlusion was performed for $45 \mathrm{~min}$. Then, $10 \mathrm{mg} / \mathrm{Kg}$ peptides were injected through the tail vein. The ligature was then removed, and the animals were maintained for 1 week of reperfusion. The mice were killed by inhalation of $25 \% \mathrm{CO}_{2}$, until respiratory and cardiac arrest occurred. Then, 2,3,5-triphenyltetrazolium chloride (TTC) and Evans blue staining or tissue harvesting for histological staining were performed to analyze the infarct size and fibrosis rates. Plasma was collected to measure cTnI and creatine kinase (CK)-MB levels. All animal studies were approved by the Animal Care and Ethics Committee of Nanjing Medical University. The animal experiments were performed according to the guide for the Care and Use of Laboratory Animals.

\section{TTC + Evans blue}

After 1 week of reperfusion, Evans blue and TTC dyes were used to determine the infarct size (IS) and area at risk (AAR). The mouse was anesthetized, and LAD was re-ligated. Then $2 \mathrm{ml} 2 \%$ Evans blue was injected through right jugular vein. The heart was immediately removed and frozen and then sectioned into $1 \mathrm{~mm}$-thick slices. The slices were incubated in $1.0 \%$ TTC for $15 \mathrm{~min}$ at $37{ }^{\circ} \mathrm{C}$. Serial sections of the heart were photographed, and infarct size was determined by computerized planimetry using ImageJ software. The infarct size was then calculated and quantified.

\section{Histological analysis}

Hearts were harvested and immediately fixed in $4 \%$ paraformaldehyde for $48 \mathrm{~h}$. The samples were dehydrated, embedded in paraffin and sectioned into 5 - $\mu \mathrm{m}$-thick slices on a sliding microtome (Leica, Nussloch, Germany). Fibrosis was detected by staining paraffin-embedded sections with Masson's trichrome. Blue collagen staining was quantified using ImageJ software (version 1.52t, National Institutes of Health, Bethesda, MD, USA). The surface area of a single cardiomyocyte was measured using the Image J Program (v 1.52a, NIH, USA). The cardiomyocyte area was quantified by measuring 200 cells (randomly selected in 3 sections) in each heart with source of sample blinded. Only cells lying fully within the visual field were quantified.

\section{CK-MB, cTnI, and LDH measurement}

Mouse cTnI plasma levels were measured using an ELISA kit from Life Diagnostics, Inc. (West Chester, PA). CK-MB concentrations were measured using an ELISA kit (LSBio, Seattle, WA, USA) according to the manufacturer's protocol. The LDH concentration was also measured using an ELISA kit (Promega, Madison, WI, USA) according to the instructions. Cell supernatant and mouse serum were collected to analyze the LDH release. 


\section{DOX-induced heart failure}

Male C57BL/6J mice (6-10 weeks of age, 20 22 g) were obtained from the Model Animal Research Center of Nanjing University (Nanjing, Jiangsu, China), and all procedures were approved by the ethical committee of Nanjing Medical University. All animals were housed at $20 \sim 25{ }^{\circ} \mathrm{C}$ and $50 \sim 70 \%$ relative humidity. To induce heart failure, $5 \mathrm{mg} /$ $\mathrm{kg}$ DOX was injected for 4 consecutive weeks (i.p., on days 7, 14, 21 and 28). $10 \mathrm{mg} / \mathrm{kg}$ peptides were injected before DOX injection every time. Body weight was recorded every week. The mice were subjected to echocardiographic analysis 1 week after the last DOX injection and killed for further analysis. Mice were euthanatized by inhalation of $25 \% \mathrm{CO}_{2}$, until respiratory and cardiac arrest occurred and killed for further analysis. All animal studies were approved by the Animal Care and Ethics Committee of Nanjing Medical University.

\section{Echocardiography}

To evaluate cardiac function, mice were anesthetized with $1 \%$ isoflurane and analyzed with a Vevo 2100 High Resolution Imaging System. Cardiac contractile function in conscious, gently restrained mice was examined by echocardiography using a Vevo 2100 system (MS400C probe, Visual Sonics). Furthermore, ventricular fractional shortening (FS) was calculated as left ventricular internal diameter in diastole (LVIDd) - left ventricular internal diameter in systole (LVIDs)/LVIDd.

\section{Western blot analysis}

Proteins were isolated from cells using lysis buffer (RIPA and protease inhibitor cocktail). Protein quantification was performed using a BCA protein detection kit $(23,229$; Thermo Fisher Scientific). The same volume of protein from each sample was separated on $10 \%$ SDS-PAGE gels and transferred to nitrocellulose membranes (Millipore, Billerica, MA, USA). The membranes were blocked with 5\% milk and then incubated with primary antibodies overnight. Detailed antibody information is listed in Supple Table S3. A FluorChem M system (ProteinSimple, San Jose, CA, USA) was used to quantify bands corresponding to proteins involved in the orchestrated immune response.

\section{Real-time PCR}

Total RNA was extracted from cells using TRIzol reagent (Thermo Fisher Scientific). The concentration of RNA was determined by measuring the absorbance ratio of 260/280 nm using a NanoDrop ND-1000 spectrophotometer (Thermo Fisher Scientific). RNA was reverse transcribed using the PrimeScript ${ }^{\mathrm{TM}}$ RT Reagent Kit with gDNA Eraser (RR047A; Takara, Tokyo, Japan), and cDNA was analyzed by qRT-PCR using SYBR ${ }^{\circledR}$ Premix Ex Taq ${ }^{\mathrm{TM}}$ (RR420A; Takara, Tokyo, Japan). The data were normalized to the levels of GAPDH and further analyzed using the $2^{-\Delta \Delta C T}$ method. The sequences for all the primers used for qPCR are listed in the Supple Table S4.

\section{Biotin pull-down assay}

Biotinylated scramble and biotinylated TAG-23 peptides were synthesized by Scientific Peptide Biological Technology Co., Ltd. (Shanghai, China). The biotinylated scramble peptide was used as a control, and a total of $2 \mu \mathrm{g}$ of biotinylated peptide was added to the lysate containing Dynabeads ${ }^{\mathrm{TM}} \mathrm{M}-280$ Streptavidin Beads (Invitrogen) overnight at $4{ }^{\circ} \mathrm{C}$. Whole cell lysates were cleared by centrifugation at $12,000 \mathrm{rpm}$ for $30 \mathrm{~min}$ at $4{ }^{\circ} \mathrm{C}$ and then precleared with Dynabeads ${ }^{\mathrm{TM}} \mathrm{M}-280$ Streptavidin Beads to eliminate nonspecific binding. Then, the precleared proteins were incubated with bead-biotinylated peptide complexes overnight at $4{ }^{\circ} \mathrm{C}$. The beads were washed extensively with wash buffer $(0.1 \%$ Triton X-100, $50 \mathrm{mM}$ Tris- $\mathrm{HCl}, 300 \mathrm{mM} \mathrm{NaCl}, 5 \mathrm{mM}$ EDTA, and $0.02 \%$ sodium azide, $\mathrm{pH} 7.4$ ), and the beads/column were washed with $4 \times 1 \mathrm{~mL}$ of ice-cold buffer followed by $2 \times 1 \mathrm{ml}$ ice-cold PBS. Glycine $(0.1 \mathrm{M}, \mathrm{pH} \sim 2.5)$ was used to elute the peptide complexes. The complexes were resolved by $10 \%$ SDS-PAGE for subsequent silver staining.

\section{Silver staining and mass spectrometry}

After 10\% SDS-PAGE, the gel was maintained in a clean plastic $15-\mathrm{cm}$ dish. Silver staining was performed by strictly following the manufacturer's protocol (Pierce ${ }^{\circledR}$ Silver Stain for Mass Spectrometry, 24,600, USA). When the band was visible, the developer working solution was immediately replaced with stop solution, and the gel bands were excised for further mass spectrometry analysis (Shanghai Applied Protein Technology Co., Ltd) on a Q Exactive mass spectrometer (Proxeon Biosystems, now Thermo Fisher Scientific).

\section{RNA-seq analysis}

Total RNA was extracted using TRIzol reagent (Life Technologies, Carlsbad, CA, US) following the manufacturer's instructions, and the RNA integrity number (RIN) was evaluated using an Agilent Bioanalyzer 2100 (Agilent Technologies, Santa Clara, CA, US) to determine RNA integrity. Qualified total RNA was further purified using a RNeasy mini kit (QIAGEN, GmBH, Germany) and an RNase-Free DNase Set (QIAGEN, GmBH, Germany). Total RNA was amplified and labeled with the Low Input 
Quick Amp Labeling Kit, One-Color (Agilent Technologies, Santa Clara, CA, US). Data were extracted with TapeStation Analysis Software A.02.01 SR1.

\section{Immunoprecipitation}

For ubiquitination assay, different plasmids were transfected to $\mathrm{H} 293$ or $\mathrm{H} 9 \mathrm{C} 2$ cells. After $48 \mathrm{~h}$ transfection, $10 \mu \mathrm{m}$ MG132 was pretreatment for $2 \mathrm{~h}$ and then cells were collected for further analysis. Cells were lysed at $4{ }^{\circ} \mathrm{C}$ using lysis buffer (50 mM Tris- $\mathrm{HCl}, 150 \mathrm{mM} \mathrm{NaCl}, 1 \mathrm{mM}$ EDTA, $10 \mathrm{mM} \mathrm{NaF}, 1 \mathrm{mM}$ sodium vanadate, and $0.5 \% \mathrm{NP}-40, \mathrm{pH}$ $7.5)$ containing protease inhibitor cocktail (Roche). Whole cell lysates were cleared by centrifugation at $12,000 \mathrm{rpm}$ for $30 \mathrm{~min}$ at $4{ }^{\circ} \mathrm{C}$ and then precleared with protein $\mathrm{A} / \mathrm{G}-$ Magnetic Beads (Thermo Scientific Fisher) to eliminate nonspecific binding. The indicated antibodies and Protein A/G-Magnetic Beads (Thermo Scientific Fisher) were incubated overnight at $4{ }^{\circ} \mathrm{C}$. Then, the precleared proteins were incubated with bead-antibody complexes overnight at $4{ }^{\circ} \mathrm{C}$. The beads were washed extensively with wash buffer (0.1\% Triton X-100, $50 \mathrm{mM}$ Tris- $\mathrm{HCl}, 300 \mathrm{mM} \mathrm{NaCl}, 5 \mathrm{mM}$ EDTA, and $0.02 \%$ sodium azide, $\mathrm{pH} 7.4$ ), and the beads/ column were washed $4 \times 1 \mathrm{~mL}$ of ice-cold buffer followed by $2 \times 1 \mathrm{ml}$ ice-cold PBS. The proteins were resolved by $8-12 \%$ SDS-PAGE for subsequent western blotting.

\section{Statistical analysis}

The data are expressed as the mean \pm SD unless otherwise indicated. Statistical differences were analyzed by unpaired 2-sided Student's $t$ test or 2-way ANOVA with Bonferroni correction for multiple comparisons. Data analysis was performed with GraphPad Prism software version 8.0. A value of $P \leq 0.05$ was considered statistically significant.

\section{Results}

\section{Function of TAG-23 in cardiomyocytes exposed to $H / R$}

To study the function of TAG-23 peptide, we first constructed an H/R model which is a well-established model for studying ischemia reperfusion injury [35]. Z-VAD, an apoptosis inhibitor, served as a positive control [14]. Cell viability was significantly reduced after $\mathrm{H} / \mathrm{R}$ treatment, and Z-VAD restored apoptosis (Supple Fig. 1A). To explore the localization of TAG-23 peptide, we synthesized FITClabeled TAG-23 and added it to the culture medium for $2 \mathrm{~h}$. The results showed that TAG-23 peptide was mainly located in the cytoplasm (Supple Fig. 1B). We then evaluated the cardiomyocytes toxicity through CCK8 assay. Different concentrations of TAG-23 peptide had no influence under normoxic conditions (Supple Fig. 1C). In this study, we synthesized a scramble peptide to serve as a control peptide. There was no significant difference in cell viability upon treatment with different concentrations of the scramble peptide (Supple Fig. 1D). TAG-23 blocked H/R-induced cell damage, as evidenced by cell viability and $\mathrm{LDH}$ release (Fig. 1A, B). In addition, we assessed whether different concentrations of TAG-23 can influence cell viability and LDH release. TAG-23 intervention had a dose-dependent effect (Supple Fig. 1E, F). Thus, our study is the first to report that TAG-23 may protect against hypoxia reperfusion injury.

\section{TAG-23 attenuates apoptosis and protects mitochondrial function}

To further study the function of TAG-23 in H/R injury, apoptosis was evaluated, and reductions in cleaved-caspase 3 activation, cleaved-PARP activation (Fig. 1C, D). TUNEL staining results showed that treatment of TAG-23 significantly reduced cell apoptosis (Fig. 1E, F). Notably, compared with the scramble peptide, the TAG-23 peptide reduced ROS content in the H/R model (Fig. 1G). TAG-23 significantly reduced the mitochondrial membrane potential, as evidenced by the ratio of JC-1 monomers to aggregates (Fig. 1H), which is a marker of early apoptosis. We also provided evidence that TAG- 23 protects the mitochondrial potential by measuring JC-1 fluorescence (Fig. 1I). Thus, our results revealed that TAG-23 can attenuate cell apoptosis and protect mitochondrial function.

\section{TAG-23 ameliorates MI injury}

To study the function of TAG-23 in vivo, we established a MI model. After a 45-min ischemia period, the TAG-23 peptide was injected through the tail vein. The, the animals underwent reperfusion for 1 week. The hearts were harvested for further study (Supple Fig. 2A). The infarct size was significantly decreased in the TAG-23 peptide intervention group by TTC and Evans blues assay (Fig. 2A, B). Notably, the TAG-23 group shows apoptosis reduction, as indicated by a reduction in TUNEL staining (Fig. 2C, D). MI-induced injury was profoundly ameliorated in the TAG-23 intervention group, as evidenced by the decrease in serum cTnI and CK-MB levels (Fig. 2E). The histological images of MI tissue showed disorganized myocardial fibers and disarrayed cardiomyocytes; however, TAG-23 intervention maintained the shape of myocardial fibers and less neutrophil infiltration (Fig. 2F). The fibrosis rate, which was detected by Masson staining, revealed that cardiac fibrosis was significantly suppressed by TAG-23 (Fig. 2G, H). These results revealed that TAG-23 plays a cardioprotective role in vivo in MI. 
A

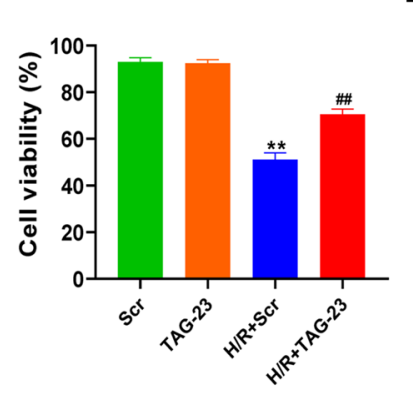

E
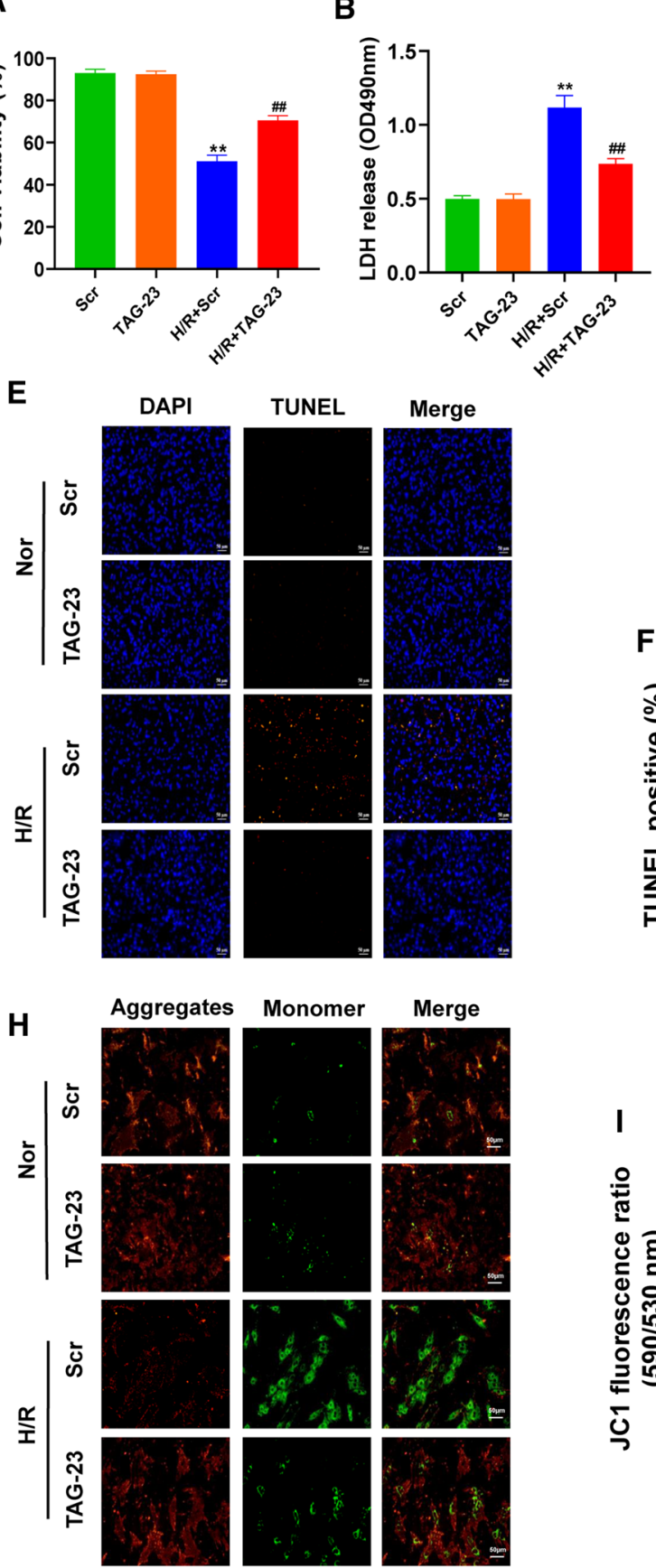

C
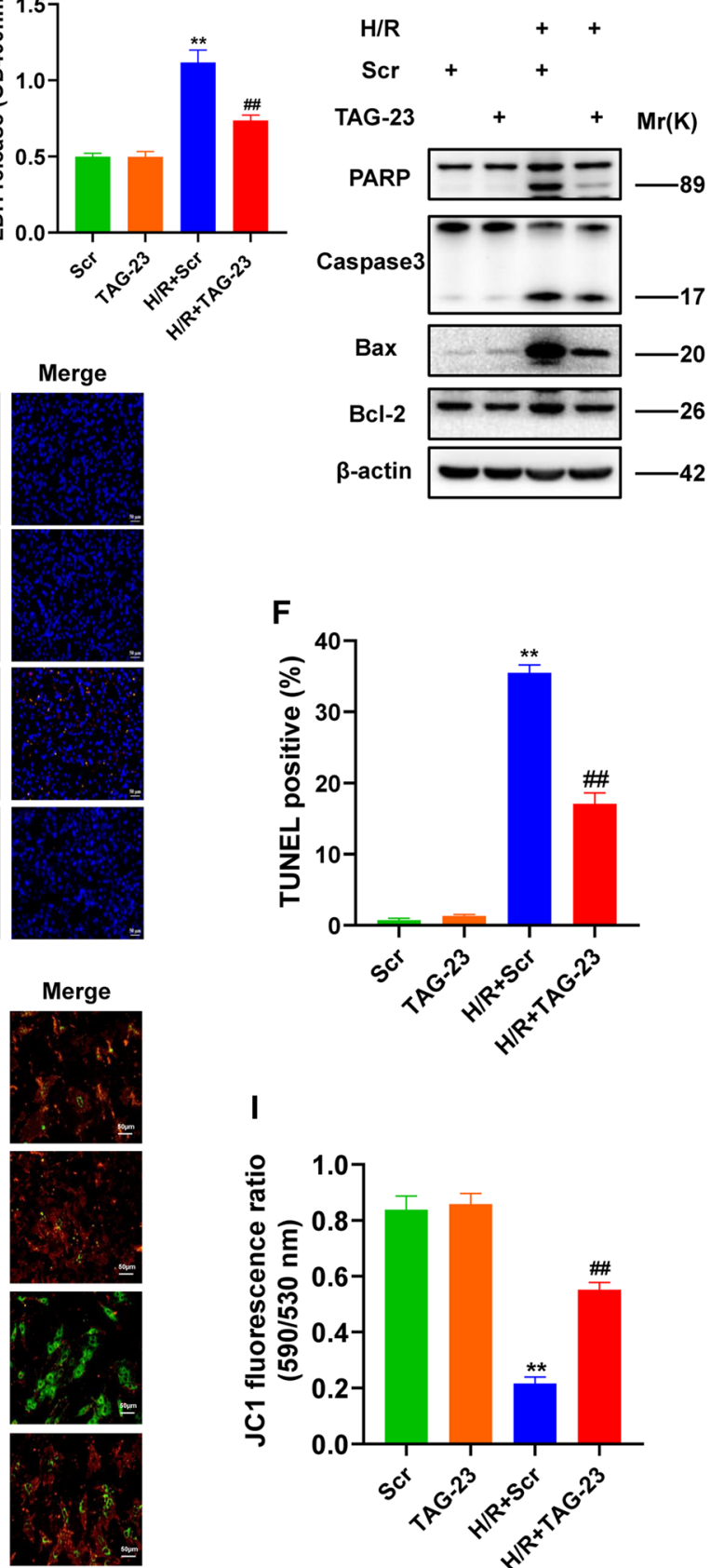

$\mathbf{F}$
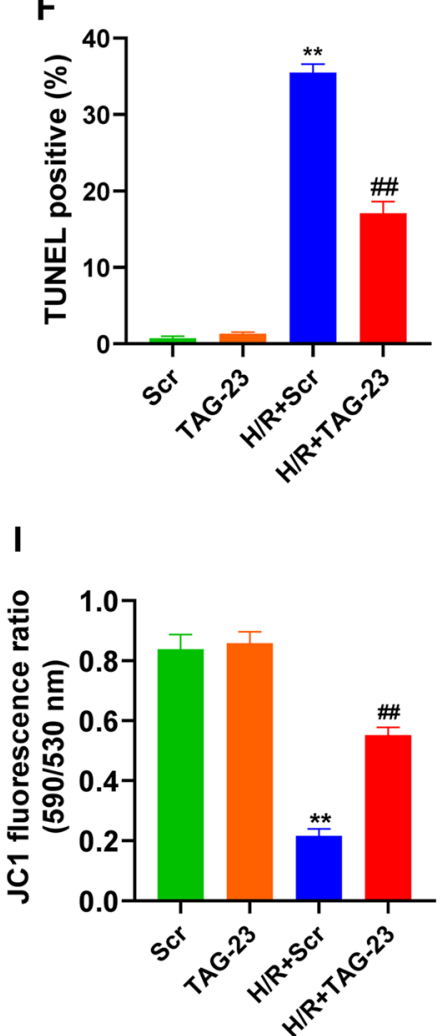

D

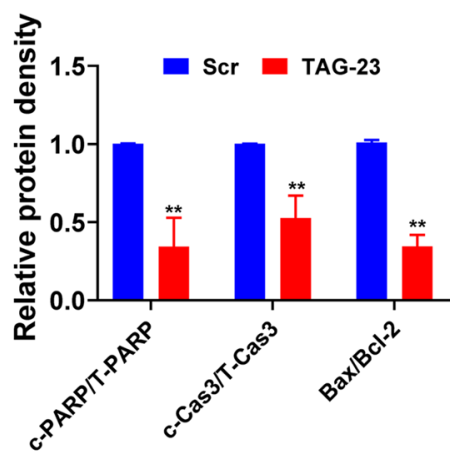

G

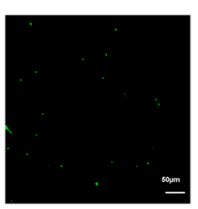

Scr

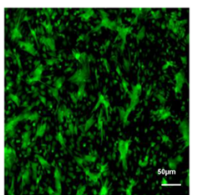

$\mathrm{H} / \mathrm{R}+\mathrm{Scr}$

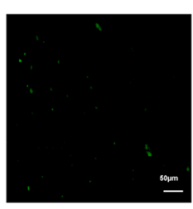

TAG-23

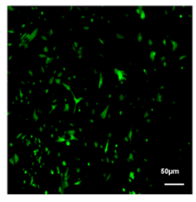

H/R+TAG-23

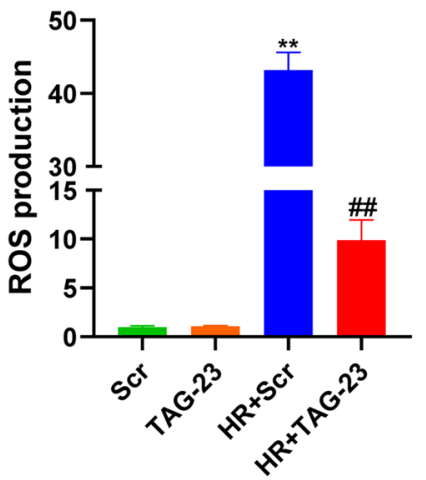

Fig. 1 Function of TAG-23 in cardiomyocytes exposed to I/R. A The cell viability was measured in H/R model. TAG-23 increased the cell viability compared with scramble peptide group. $n=3$ per group (two-way ANOVA analysis with Bonferroni's multiple comparison test). B LDH release was measured in H/R model. TAG-23 reduced the LDH release compared with scramble peptide group. $n=3$ per group (two-way ANOVA analysis with Bonferroni's multiple comparison test). C Apoptosis markers (PARP, Caspase3, Bax, Bcl-2) were measured by western blot. TAG-23 significantly inhibits H/R-induced cell apoptosis. D Quantification data of western blot in H/R group. $n=3$ per group (Student's $t$ test). E Representative pho- tography of TUNEL assay. F Quantification data of TUNEL assay. TUNEL results revealed that TAG-23 inhibited the cell apoptosis induced by $\mathrm{H} / \mathrm{R}$ exposure. $n=3$ per group (two-way ANOVA analysis with Bonferroni's multiple comparison test). G Representative photography of ROS contents (up) and quantification data (down). $n=3$ per group (two-way ANOVA analysis with Bonferroni's multiple comparison test). H Representative photography of mitochondrial membrane potential in $\mathrm{H} / \mathrm{R}$ model. I JC-1 was measured under $590 / 530 \mathrm{~nm} . n=3$ per group (two-way ANOVA analysis with Bonferroni's multiple comparison test). $* * P<0.01, * * * P<0.001$. Data are means \pm SD with $n=3$ independent biological cultures 
A $\mathrm{Ml}+\mathrm{Scr}$
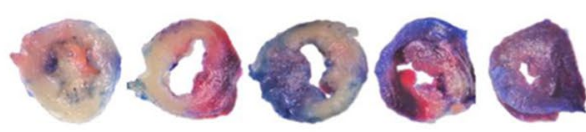

MI+TAG-23
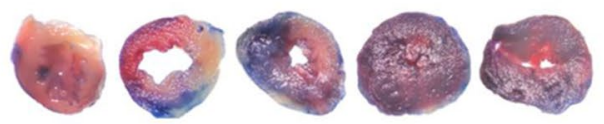

D

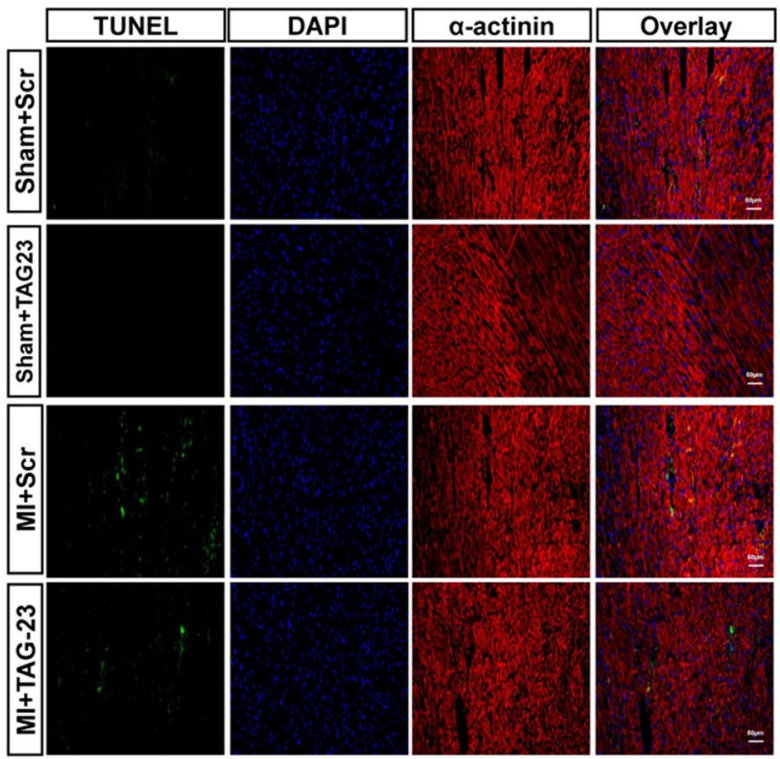

B

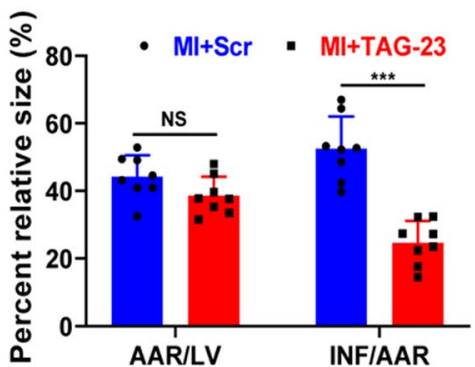

C

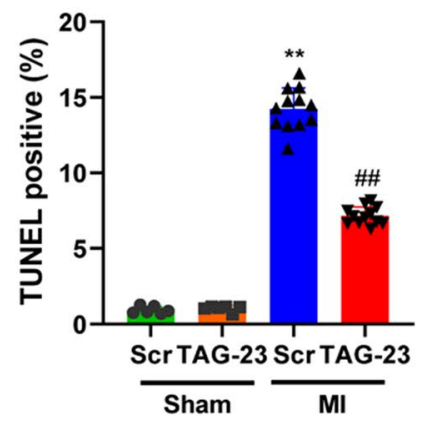

E
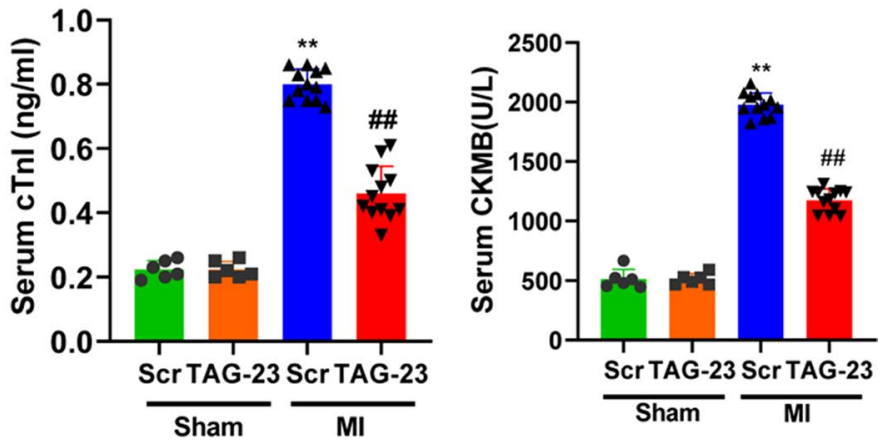

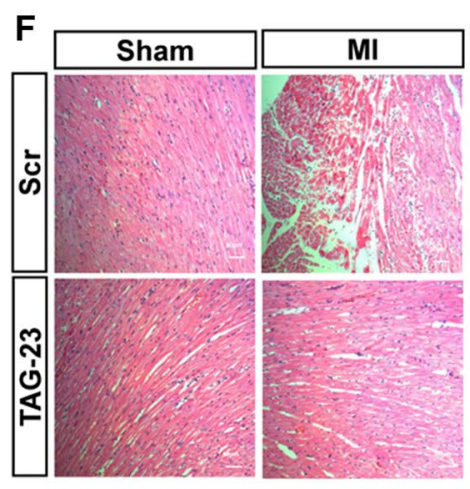

G

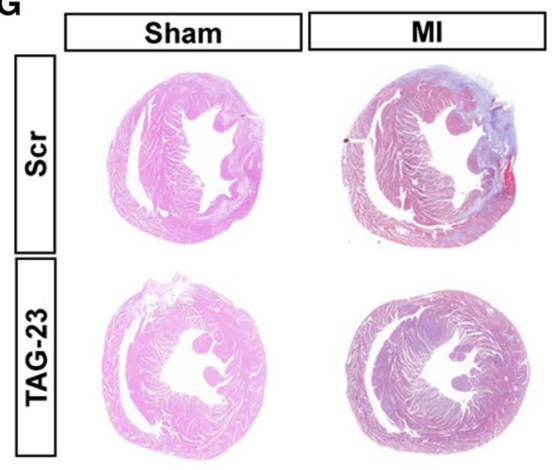

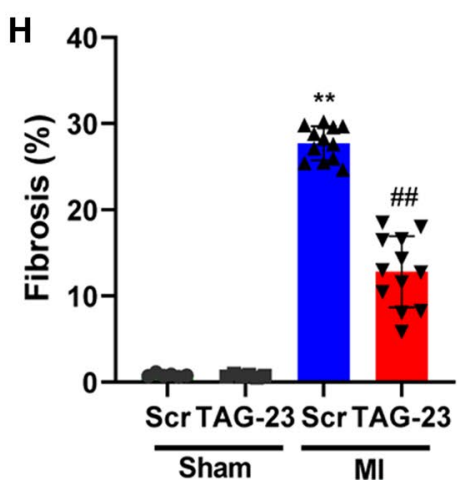

Fig. 2 TAG-23 ameliorates the myocardial infarction injury. A Representative photographs of infarct size via TTC and Evans blue. B Quantification data of infarct size. $n=8$ per group (Student's $t$ test). $10 \mathrm{mg} / \mathrm{Kg}$ TAG-23 significantly reduced the infarction area compared with scramble peptide group. C Quantitative data for TUNEL staining. $n=6$ per group in sham group and $n=12$ in MI group (twoway ANOVA analysis with Bonferroni's multiple comparison test). D Representative photographs of TUNEL staining. TUNEL results demonstrated that TAG-23 inhibited cell apoptosis induced by myocardial infarction reperfusion injury. E Serum CK-MB and cTnI con- centrations measured by ELISA. $n=6$ per group in sham group and $n=12$ in MI group (two-way ANOVA analysis with Bonferroni's multiple comparison test). F Representative photographs of HE staining of heart sections. HE results showed that TAG-23 maintained the shape and size of myocardium fibers. G Representative photographs of Masson trichrome staining of heart sections. H Quantitative data for Masson staining. $n=6$ per group in sham group and $n=12$ in MI group (two-way ANOVA analysis with Bonferroni's multiple comparison test). $* * P<0.01, * * * P<0.001$. Data are means \pm SD with $n=6 / 12$ independent biological replicates 


\section{TAG-23 attenuates DOX-induced heart failure}

Previous studies have reported that DOX, a chemotherapeutic agent, can also trigger excessive loss of cardiomyocytes and lead to heart failure. Increasing evidence has demonstrated that oxidative stress is, at least in part, responsible for the DOX-induced cardiac injury. TAG-23 was injected through the tail vein every time before DOX treatment [40]. DOX $(5 \mathrm{mg} / \mathrm{kg})$ was intraperitoneally injected for 4 consecutive weeks to induce cardiomyocyte loss (Supple Fig. 2B).
DOX-induced cardiac function was remarkably decreased in the scramble + DOX group; however, TAG-23 intervention significantly attenuated the echocardiographic phenotype (Fig. 3A). DOX-induced cardiac contractile dysfunction, which was indicated by decreases in ejection fraction (EF) and FS, and ventricular systolic dysfunction, which was indicated by the left ventricular end-systolic dimension (LVESd), were effectively improved in the TAG-23 injection group (Fig. 3B). However, there was no significant difference in left ventricular end-diastolic dimension (LVEDd) or
A

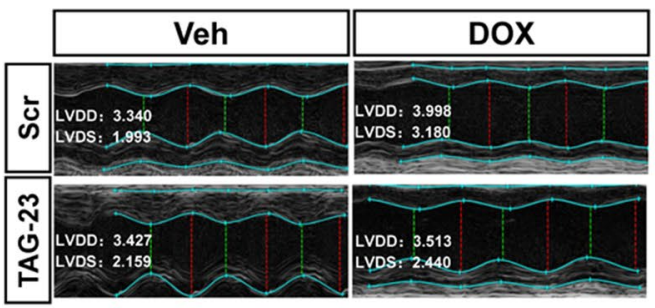

C
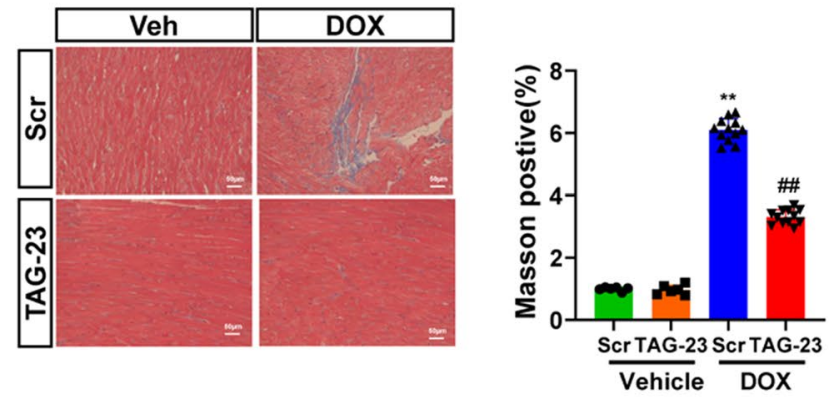

E

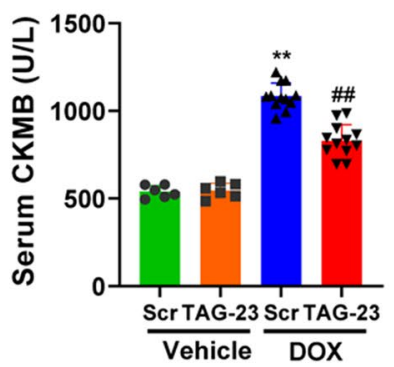

$\mathbf{F}$

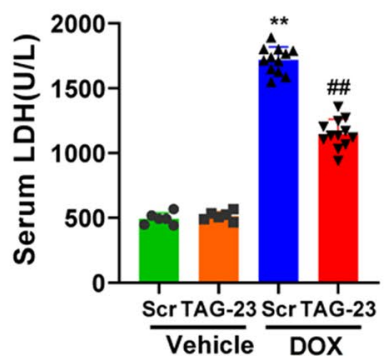

Fig. 3 TAG-23 attenuates the DOX-induced heart failure. A Representative photographs of echocardiography of DOX-induced cardiac damage. TAG-23 maintained the cardiac function after DOX injection. B Quantitative data for echocardiography ( $n=6$ mice per group in vehicle group and $n=12$ in DOX group, two-way ANOVA analysis with Bonferroni's multiple comparison test, EF, FS, LVEDs). C Representative photographs of Masson trichrome staining of heart sections (left) and quantification data (right). $n=6$ mice per group in vehicle group and $n=12$ in DOX group (two-way ANOVA analysis with Bonferroni's multiple comparison test). D Representative photographs of TUNEL staining (left) and quantification data (right). $n=6$ mice per group in vehicle group and $n=12$ in DOX group (two-way

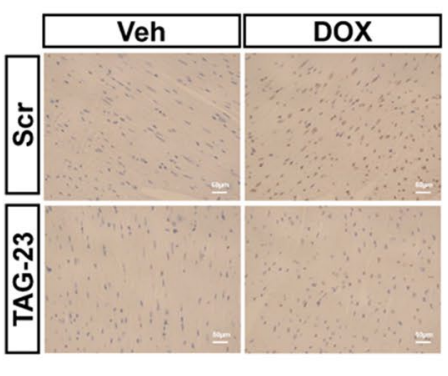

G

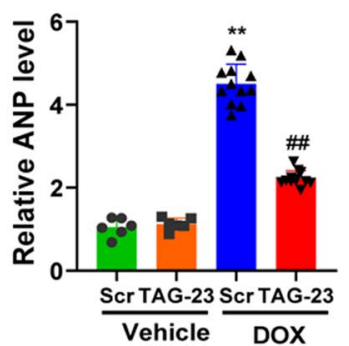

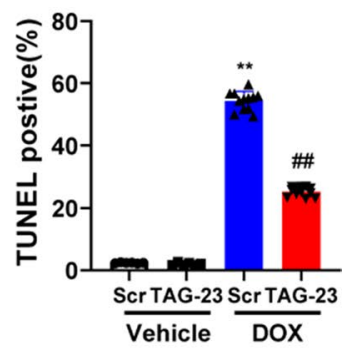

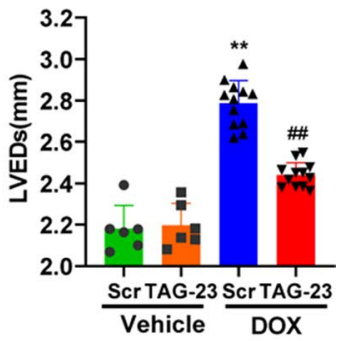

H

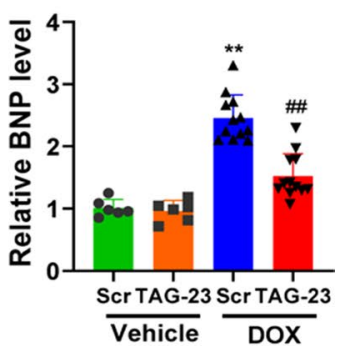

ANOVA analysis with Bonferroni's multiple comparison test). E Serum CK-MB concentrations measured by ELISA. $n=6$ mice per group in vehicle group and $n=12$ in DOX group (two-way ANOVA analysis with Bonferroni's multiple comparison test). F Serum LDH concentrations measured by LDH kit. $n=6$ mice per group in vehicle group and $n=12$ in DOX group (two-way ANOVA analysis with Bonferroni's multiple comparison test) G-H ANP and BNP mRNA level was measured via qPCR. $n=6$ mice per group in vehicle group and $n=12$ in DOX group (two-way ANOVA analysis with Bonferroni's multiple comparison test). $* * P<0.01$, $* * * P<0.001$. Data are means \pm SD with $n=6 / 12$ independent biological replicates 
A

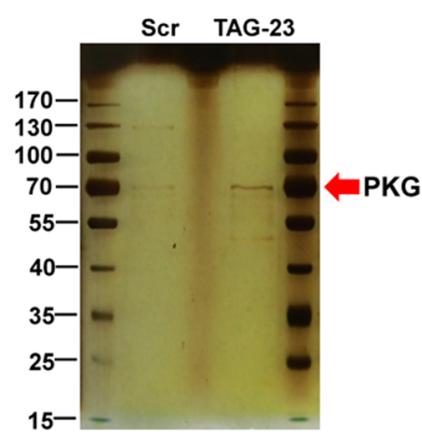

D

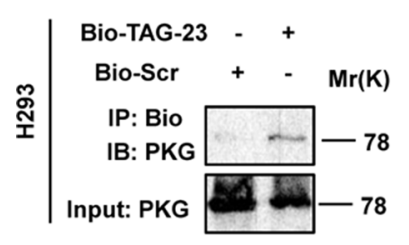

E

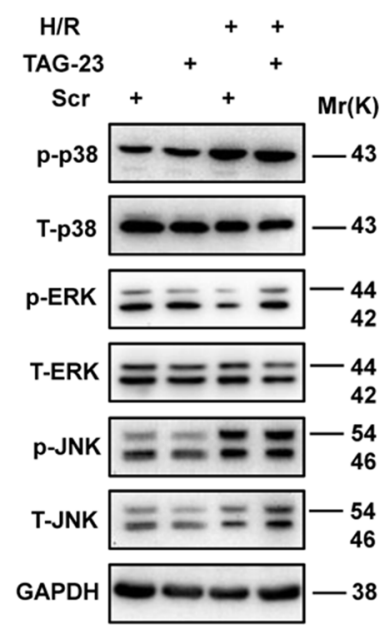

Fig. 4 TAG-23 binds to PKG to activate the ERK signaling pathway. A Representative photographs of silver staining. B Heat map of RNA-seq. $n=2$ per group, $\mathbf{c}$ KEGG analysis revealed that the top 10 pathways involved. D Interaction between TAG-23 and PKG in H293 cells and H9C2 cells. Co-IP assay was performed to verify the interaction between TAG-23 and PKG. $n=3$ per group (Student's $t$ test). E MAPK signaling pathway was verified via western blot in H/Rinduced hypoxia model. $n=3$ per group (two-way ANOVA analysis with Bonferroni's multiple comparison test). F MAPK signaling
C

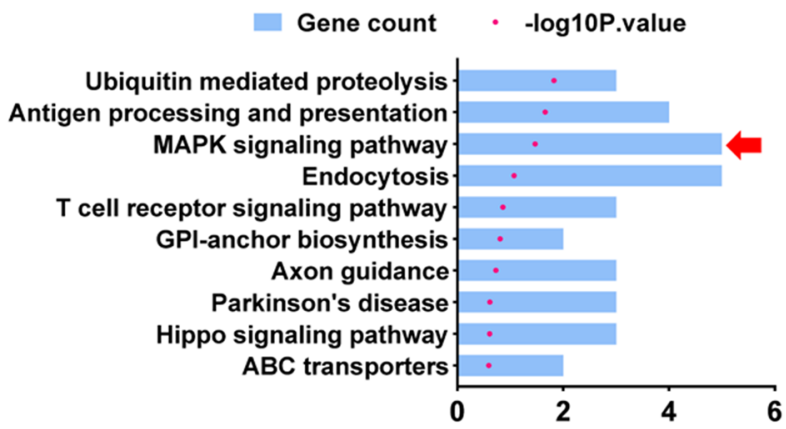

G
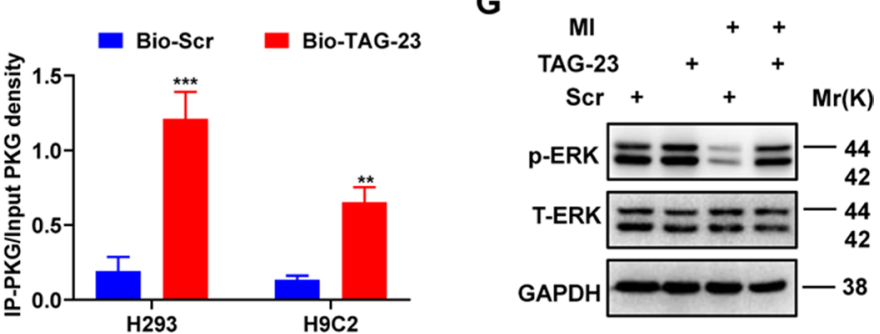

$\mathbf{F}$
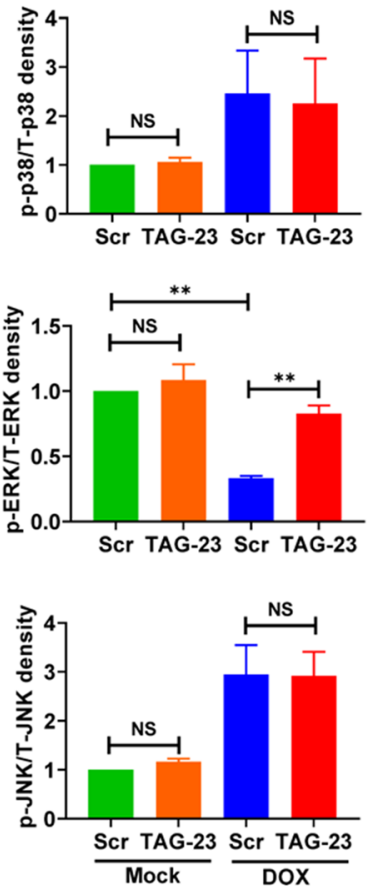

pathway was verified via western blot in DOX-induced oxidative stress. $n=3$ per group (two-way ANOVA analysis with Bonferroni's multiple comparison test). G ERK signaling pathway was verified via western blot in myocardial infarction tissues. TAG-23 significantly increased the ERK signaling pathway compared with scramble peptide group. $n=3$ per group (two-way ANOVA analysis with Bonferroni's multiple comparison test). $* * P<0.01, * * * P<0.001$. Data are means \pm SD with $n=3$ independent biological cultures 
left ventricular posterior wall thickness (LVPWd) (Supple Fig. 2C, Supple Table S1). Body weight was significantly decreased in the DOX injection group, whereas TAG-23 abolished this effect during DOX injection (Supple Fig. 2D). Notably, TAG-23 injection protected the heart from DOXinduced alterations in cardiac morphology and injury, as evidenced by cardiac fibrosis and the cardiomyocyte area (Fig. 3C, Supple Fig. 2E). The TUNEL assay showed that the apoptosis rates were decreased in the TAG-23 group, suggesting that TAG-23 exerted an antiapoptotic effect (Fig. 3D). DOX-induced cardiomyocyte damage was profoundly ameliorated in the TAG-23 intervention group, as indicated by decreased concentrations of serum CK-MB and LDH levels (Fig. 3E, F). Finally, we measured the mRNA levels of the cardiac injury markers atrial natriuretic peptide (ANP) and B-type natriuretic peptide (BNP), which were significantly attenuated in the TAG-23 injection group compared with the scramble peptide injection group (Fig. 3G, $\mathrm{H})$. These data demonstrated that, similar to its role in MI, TAG-23 also plays a protective role in DOX-induced cardiac damage and heart failure.

\section{TAG-23 binds to PKG to activate the ERK signaling pathway}

To investigate the mechanism of TAG-23 peptide involvement, we first performed a biotin pull-down assay. Due to the affinity of the peptide itself, we acquired a significant band that was approximately $70 \sim 80 \mathrm{kDa}$ (Fig. 4A). Mass spectrometry analysis revealed the possible targets of biotinylated TAG-23, and the top 30 enriched proteins are listed in Supple Table S2. Among those enriched proteins, PKG, which has already been shown to protect against I/R injury, attracted our attention $[13,28]$. We also analyzed the potential pathways by RNA-seq (Fig. 4B). Interestingly, KEGG analysis showed that TAG-23 peptide may be associated with Ub-mediated proteolysis and the MAPK signaling pathway (Fig. 4C). Previous studies have shown that PKG can regulate the MAPK signaling pathway [5, 27]. Thus, we speculated that there may be a relationship between TAG-23, PKG and MAPK. First, we incubated biotinylated peptides with proteins overnight and then performed a pull-down assay with streptavidin beads. The pulled-down proteins were then subjected to western blotting. We found that PKG was pulled down by biotinylated TAG-23 peptide in HEK cells and H9C2 cells (Fig. 4D). Next, we determined the downstream pathways of PKG and whether TAG-23 treatment can influence those pathways. Previous studies have shown that p38, ERK and JNK are downstream proteins of PKG [37]. NRVMs were treated with $\mathrm{H} / \mathrm{R}$ and $\mathrm{DOX}$, respectively. TAG-23 had no effect on p-p38 or p-JNK, and the total protein levels of p38 and JNK was unchanged (Fig. 4E, F, lane 3 vs. lane 4). However, there was a significant increase in the
Fig. 5 TAG-23 protects cardiomyocytes from reperfusion injury through ERK signaling pathway. A ERK inhibitor rescued the cell viability phenotype of TAG-23. $n=3$ per group (one-way ANOVA analysis with Bonferroni's multiple comparison test). B ERK inhibitor rescued the LDH release of TAG-23. $n=3$ per group (one-way ANOVA analysis with Bonferroni's multiple comparison test). C $20 \mu \mathrm{m}$ ERK inhibitor PD98059 was utilized to inhibit the activation of ERK. D Quantification data of western blot results of rescue experiment. $n=3$ per group (one-way ANOVA analysis with Bonferroni's multiple comparison test). E Representative photography of TUNEL assay. F Quantification data of TUNEL assay. ERK inhibitor rescued the TAG-23 phenotype in H/R model. $n=3$ per group (one-way ANOVA analysis with Bonferroni's multiple comparison test). G Representative photography of ROS contents (up) and quantification data (down). $n=3$ per group (one-way ANOVA analysis with Bonferroni's multiple comparison test). $\mathbf{H}$ Representative photography of mitochondrial membrane potential in different treatment. I JC-1 was measured under $590 / 530 \mathrm{~nm} . n=3$ per group (one-way ANOVA analysis with Bonferroni's multiple comparison test). $* * P<0.01$, $* * * P<0.001$. Data are means \pm SD with $n=3$ independent biological cultures

p-ERK level after TAG-23 intervention, whereas the level of p-ERK was notably downregulated in the scramble peptide group (Fig. 4E, F lane 3 vs. lane 4). We also verified the ERK signaling pathway in myocardial infarction tissues. TAG-23 can activate the p-ERK to protect against myocardial reperfusion injury (Fig. 4G).

\section{TAG-23 protects cardiomyocytes from reperfusion injury through ERK signaling pathway}

Previous studies have shown that p-ERK can protect against $\mathrm{H} / \mathrm{R}$ injury by inhibiting apoptosis and oxidative stress [3, 6]. We wondered whether TAG-23 protect cardiomyocytes against reperfusion injury through ERK signaling pathway. Thus, ERK inhibitor, PD98059, was utilized to confirm the signaling pathway was inhibited. The cell damage was rescued by treatment with PD98059 and TAG-23, as indicated in cell viability and LDH release (Fig. 5A, B). Cell apoptosis was evaluated by western blot and TUNEL assay (Fig. 5C-F), which indicated that decreased apoptosis rates in TAG-23 group and increased apoptosis rates in TAG-23 and PD98059 group. Furthermore, PD98059 also restored the effect of mitochondrial membrane potential and ROS contents which was induced by TAG-23 (Fig. 5H-I). Thus, our results demonstrated that TAG-23 can bind with PKG and activate the ERK signaling pathway to attenuate apoptosis and reperfusion injury.

\section{TAG-23 increases the protein level of PKG by inhibiting PKG degradation}

To explore how the peptide TAG-23 regulates $\mathrm{PKG}$ in $\mathrm{I} / \mathrm{R}$, we first analyzed the mRNA level of PKG after treatment with different concentrations of TAG-23 and at different time 


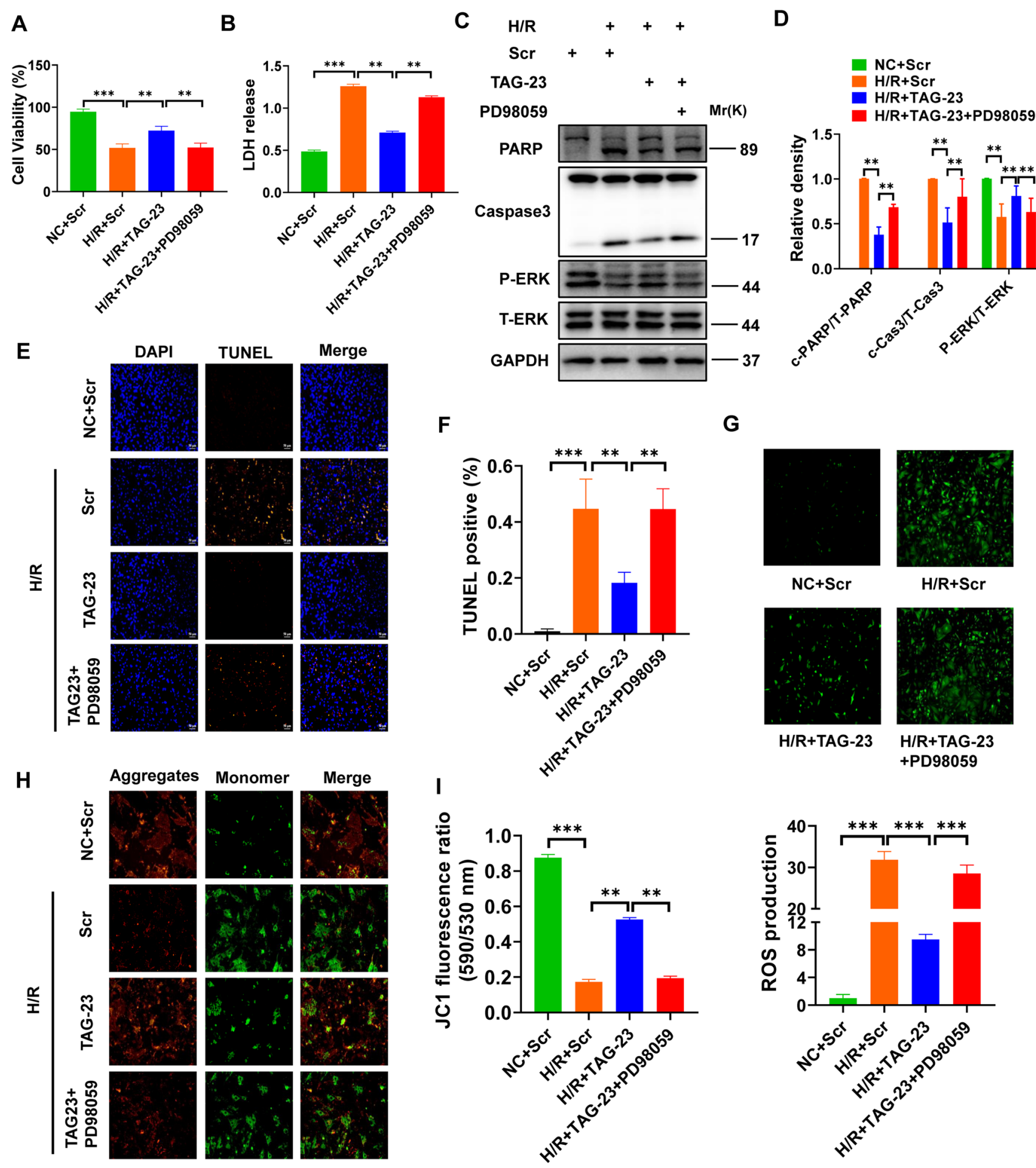

points. There was no significant difference in the mRNA level of PKG after treatment with TAG-23, suggesting that TAG-23 had no impact on the transcription of PKG (Supple Fig S1). Interestingly, the protein level of PKG was significantly increased in $\mathrm{H} 9 \mathrm{C} 2$ and $\mathrm{HEK}$ cells in a time-dependent and dose-dependent manner (Fig. 6A, B, Supple Fig. 3A, B). Besides, we also examined the P-VASP, downstream target of PKG, which was significantly upregulated upon TAG-23 treatment, suggesting that TAG-23 can activate the phosphorylation of P-VASP. These results indicated that TAG-23 may influence PKG at the posttranslational level. Previous studies have shown that PKG can be degraded through the UPS [9]. Our RNA-seq results showed that TAG-23 may be associated with Ub-mediated proteolysis (Fig. 4C); thus, 

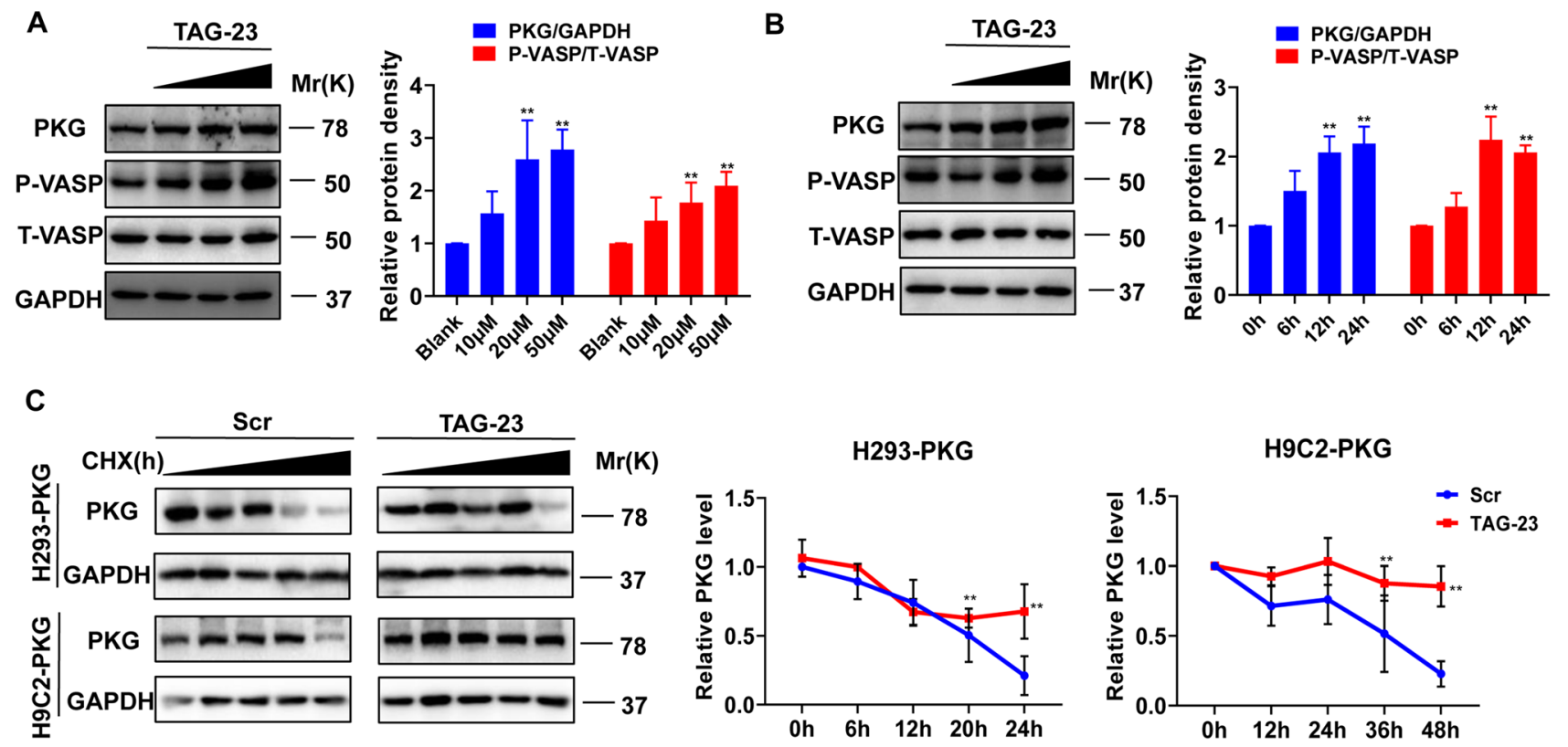

D

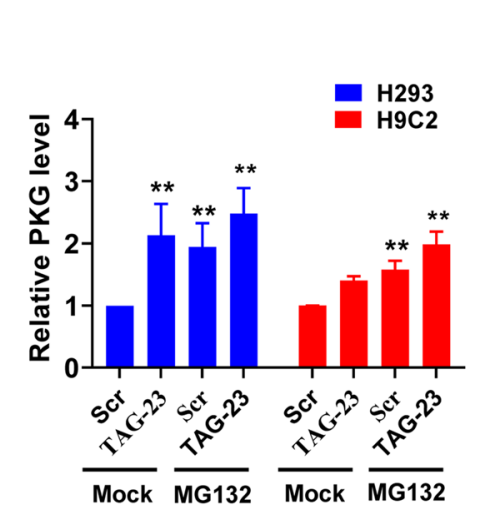

E
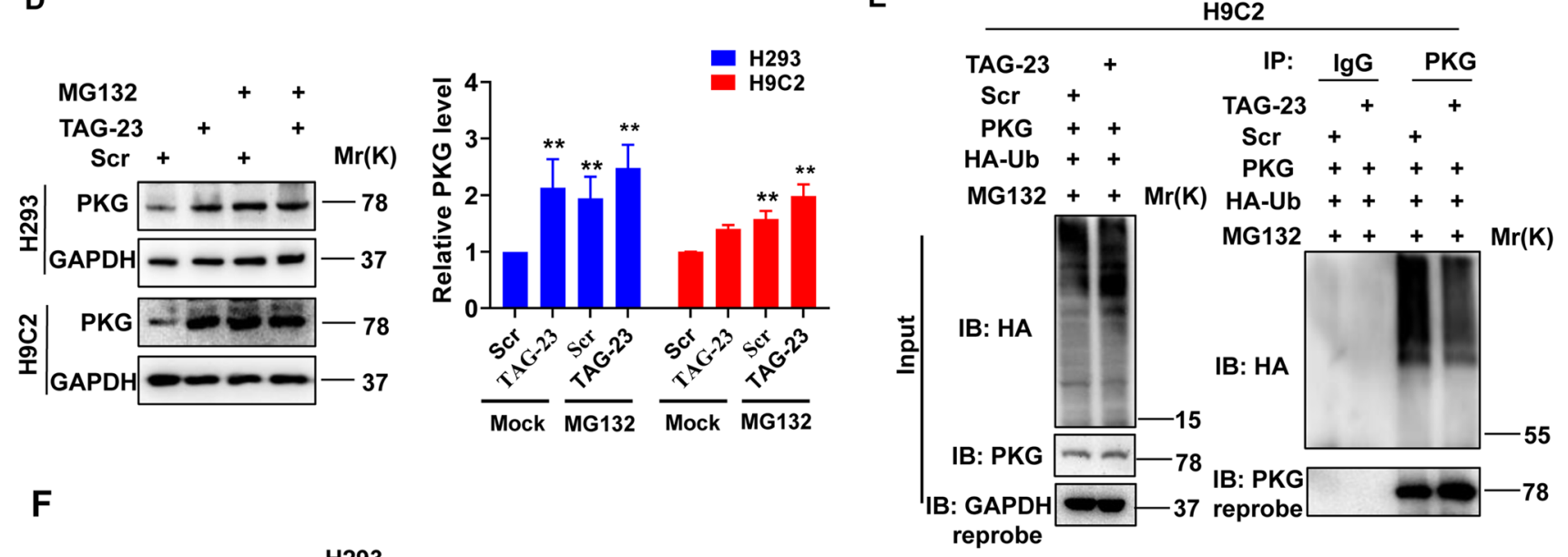

$\mathbf{F}$

H293
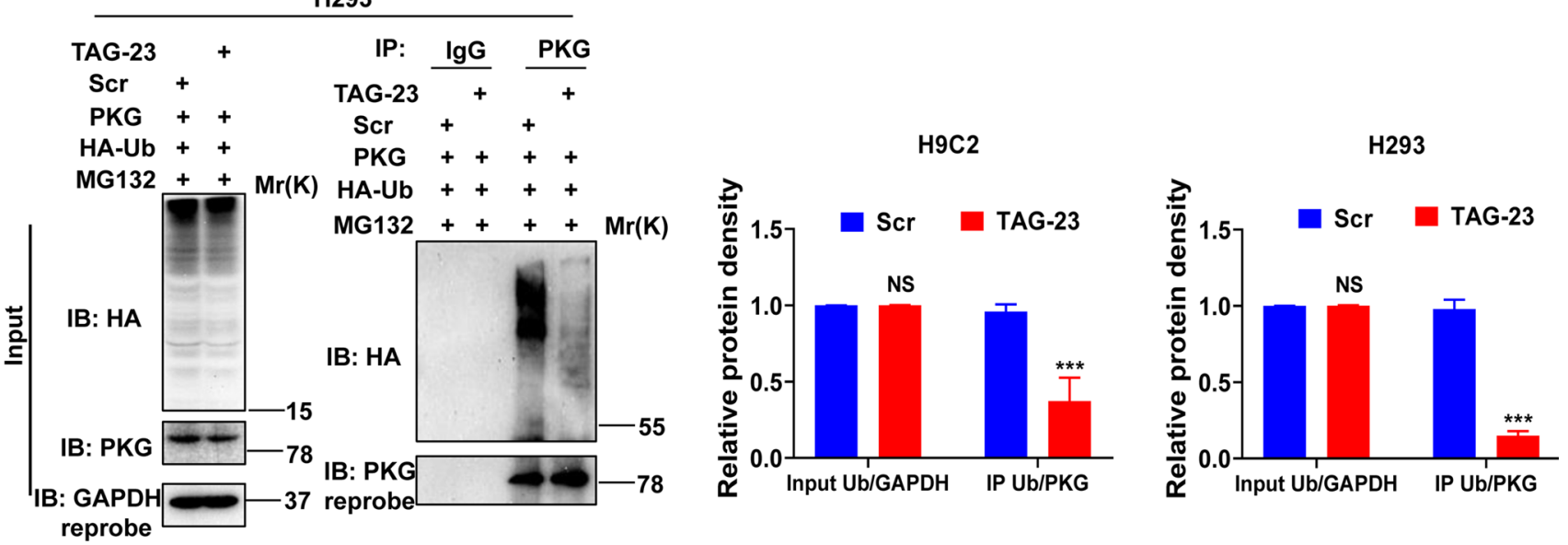

we overexpressed PKG in HEK and $\mathrm{H} 9 \mathrm{C} 2$ cells and treated them with $1 \mu \mathrm{m}$ cycloheximide (CHX) to block protein translation [10]. TAG-23 extended the half-life of PKG in HEK cells and $\mathrm{H} 9 \mathrm{C} 2$ cells. The protein level of PKG began to decrease at $48 \mathrm{~h}$ after CHX treatment, whereas TAG-23 maintained the level of PKG (Fig. 6C). We treated 
4Fig. 6 TAG-23 increases the protein level of PKG by inhibiting PKG degradation. A The protein level of PKG and P-VASP in different concentrations of TAG-23 $(10,20,50 \mu \mathrm{M})$ in H9C2 cells. TAG-23 increased the PKG protein level in a dose-dependent manner. $n=3$ per group (linear regression analysis). B The protein level of PKG and P-VASP in different time points $(0,6,12,24 \mathrm{~h})$ in $\mathrm{H} 9 \mathrm{C} 2$ cells. TAG-23 increased the PKG protein level in a time-dependent manner. $n=3$ per group (linear regression analysis). C $1 \mu \mathrm{m}$ CHX was used to detect the half-life of PKG in H293-PKG cells (up, 0, 6, 12, 18, $24 \mathrm{~h}$ ) and H9C2-PKG cells (down, 0, 12, 24, 36, $48 \mathrm{~h}$ ). TAG-23 extended the half-life of PKG in two different cell lines. Quantitative data for western blot (right). $n=3$ per group (Student's $t$ test). D The protein level of PKG treated with $10 \mu \mathrm{m}$ MG132 and $50 \mu \mathrm{m}$ TAG-23 for 2 h. $n=3$ per group (two-way ANOVA analysis with Bonferroni's multiple comparison test). $\mathbf{E}$ The ubiquitination of PKG was detected in $\mathrm{H} 9 \mathrm{C} 2$ cells. TAG-23 inhibited the PKG ubiquitination of PKG in H9C2 cells. $n=3$ per group (Student's $t$ test). F The ubiquitination of PKG was detected in $\mathrm{H} 293$ cells. TAG-23 inhibited the PKG ubiquitination of PKG in $\mathrm{H} 293$ cells. $n=3$ per group (Student's $t$ test). $* * P<0.01, * * * P<0.001$. Data are means \pm SD with $n=3$ independent biological replicates

cells with MG132, a potent, reversible peptide aldehyde, which can effectively block the proteolytic activity of proteasome complex, to detect whether PKG degradation is influenced by the Ub system. The protein level of PKG was significantly increased after treatment with $10 \mu \mathrm{m}$ MG132 for $2 \mathrm{~h}$ (Fig. 6D, lane 3 vs. lane 1). Finally, we detected whether TAG-23 can influence the ubiquitination of PKG. HA-Ub and PKG were cotransfected into H9C2 and HEK cells. After $10 \mu \mathrm{m}$ MG132 treatment for $2 \mathrm{~h}$, proteins were collected to detect ubiquitination. The total ubiquitination of proteins was unchanged. However, compared with the scramble peptide, TAG-23 significantly inhibited the ubiquitination of PKG in $\mathrm{H} 9 \mathrm{C} 2$ cells and HEK cells after it was pulled down with PKG (Fig. 6E, F). Thus, our results demonstrated that TAG-23 can regulate the protein level of PKG at the posttranslational level by inhibiting the degradation of PKG through the UPS.

\section{TAG-23 inhibits PKG degradation by attenuating the PKG-cCbl interaction}

To date, there have been no reports on the E3 ligase of PKG. Therefore, we first performed a pull-down assay (Supple Fig S2). Our silver staining results showed more bands in the PKG group than the IgG group. The significant bands were incised for mass spectrometry analysis. We found that 4 E3 ligases, cCbl, RNF13, HUWE1, and PDZRN3, were enriched in the isolated bands. We cotransfected vectors overexpressing these E3 ligases and PKG in HEK293 cells. Our results showed that cCbl was pulled down by PKG in HEK cells and H9C2 cells (Fig. 7A, B). To the best of our knowledge, this is the first report on the E3 ligase of PKG. cCbl overexpression effectively decreased the protein level of PKG in HEK cells and H9C2 cells (Fig. 7C, D). To further demonstrate that $\mathrm{cCbl}$ is the $\mathrm{E} 3$ ligase of $\mathrm{PKG}$, we cotransfected cCbl, PKG and HA-Ub plasmids into $\mathrm{H} 293$ cells. After $72 \mathrm{~h}$ of transfection, HEK cells were treated with $10 \mu \mathrm{m} \mathrm{MG132}$ for $2 \mathrm{~h}$. We found that cCbl overexpression promoted the ubiquitination of PKG (Fig. 7E, F, lane 2 vs. lane 1).

We speculated that there may be a relationship between peptide TAG-23, cCbl and PKG. Our results demonstrated that TAG-23 treatment noticeably reduced the protein level of PKG after PKG and cCbl overexpression, suggesting that TAG-23 can serve as a competitive peptide to attenuate the PKG-cCbl interaction (Fig. 7G, H). To further verify these results, we cotransfected cCbl, PKG and Ub into H9C2 cells. After $72 \mathrm{~h}$ transfection, cells were treated with or without TAG-23. cCbl overexpression significantly promoted the ubiquitination of PKG (Fig. 7I, lane 2 vs. lane 1). Treatment with TAG-23 peptide reduced the ubiquitination of PKG (Fig. 7I, lane 4 vs. lane 2). Similar results can be achieved in H293 cells (Supple Fig. 3A, B). Taken together, our data provide comprehensive evidence that $\mathrm{cCbl}$ is the $\mathrm{E} 3$ ligase of PKG and that the PKG-cCbl interaction is impaired by TAG-23 treatment (Fig. 8).

\section{TAG-23 mediates PKG degradation at the Lys48 site}

To further identify the possible ubiquitination sites, we constructed different mutant Ub plasmids (WT, KO, K48R, K63R). PKG and the mutant Ub plasmid were cotransfected into $\mathrm{H} 9 \mathrm{C} 2$ cells. After $72 \mathrm{~h}$ of culture, the H9C2 cells were treated with TAG-23 and MG132 for 2 h. Our results suggested that little ubiquitination occurred without Ub overexpression (Fig. 7J, lane 2 vs. lane 1). Treatment with TAG-23 significantly inhibited ubiquitination in the WT-Ub and PKG groups (Fig. 7J, lane 3 vs. lane 4). However, there was no difference in the KO group with or without TAG-23 treatment (Fig. 7J, lane 6 vs. lane 5). Interestingly, there was still a significant difference in the K63R mutant Ub group, suggesting that the K63R mutant had no impact on the ubiquitination of PKG (Fig. 7J, lane 9 vs. lane 10). We also verified above results in $\mathrm{H} 293$ cells (Supple Fig. 3E, F). These effects suggested that TAG-23 mediates PKG degradation at the Lys48 site.

\section{Discussion}

Here, we demonstrate that peptide TAG-23 has a therapeutic role in reperfusion and oxidative stress, which result in MI and heart failure. First, TAG-23 peptide was shown to be efficiently internalized in cardiomyocytes and reduce cell apoptosis, ROS content, and oxidative stress in vitro. In vivo, TAG-23 peptide significantly reduced the infarct size in a MI model. Moreover, we demonstrated that TAG-23 can inhibit 
A
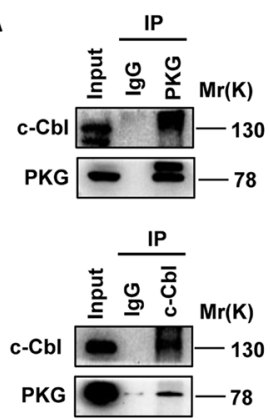
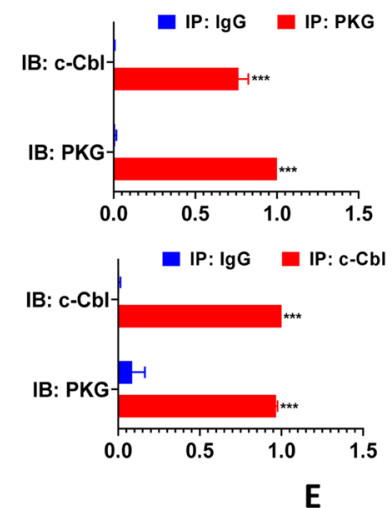

C

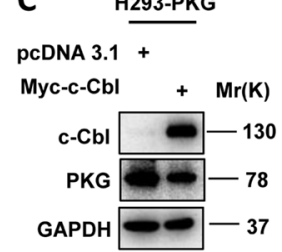

D

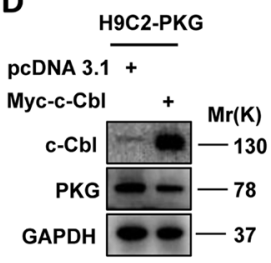

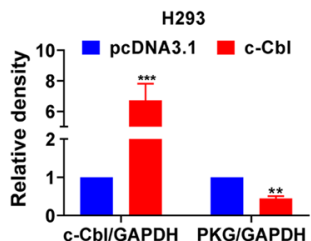

E
B
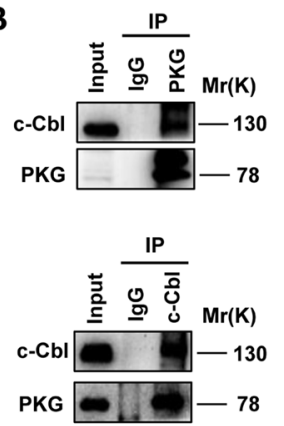
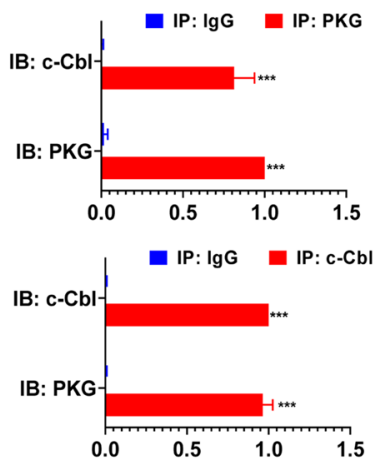

G
$\mathbf{F}$

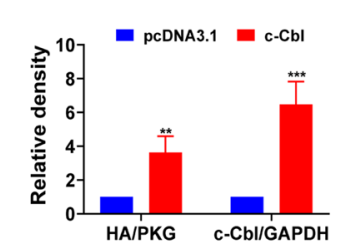

H
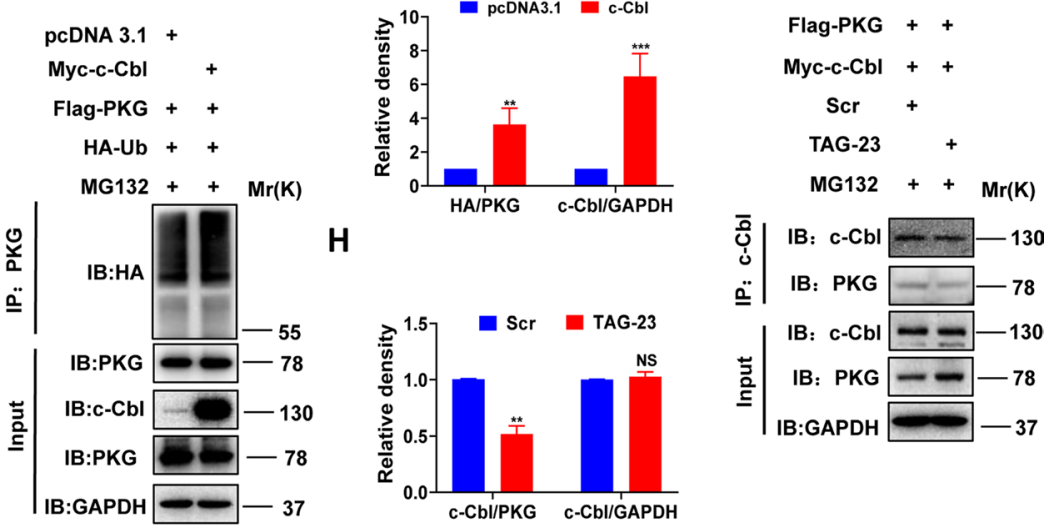

I

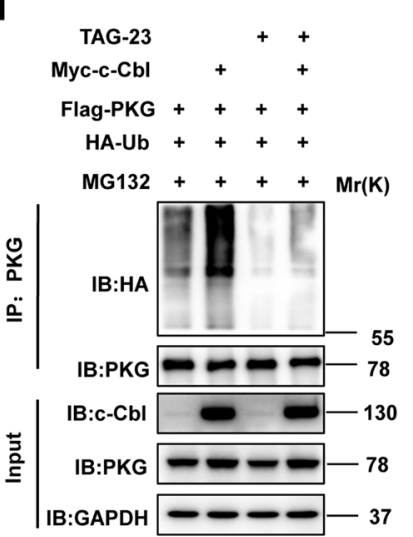

J

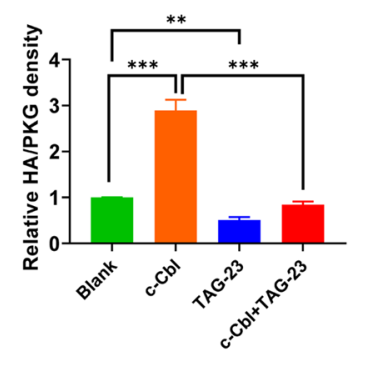

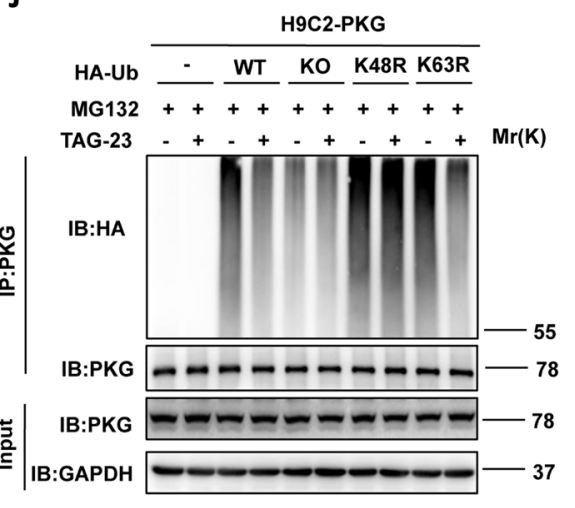

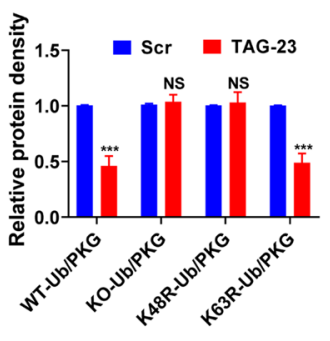

Fig. 7 TAG-23 inhibits PKG degradation by attenuating PKG-cCbl interaction. A The interaction between PKG and cCbl in H293 cells was verified by Co-IP. $n=3$ per group (Student's $t$ test). B PKG-cCbl interaction was verified in H9C2 cells via Co-IP. $n=3$ per group (Student's $t$ test). C Overexpression of cCbl leads to the decrease of protein level of PKG in H293 cells. $n=3$ per group (Student's $t$ test). D Overexpression of cCbl leads to the decrease of protein level of PKG in H9C2 cells. $n=3$ per group (Student's $t$ test). E Overexpression of cCbl promotes the ubiquitination of PKG in H293 cells. Cells were treated with $10 \mu \mathrm{m} \mathrm{MG132}$ for $2 \mathrm{~h}$. F Quantification data of western blot results of ubiquitination. $n=3$ per group (Student's $t$ test). $\mathbf{G}$
TAG-23 attenuates the PKG-cCbl interaction in H9C2 cells. TAG-23 can serve as a competitive peptide to attenuate the cCbl-PKG interaction. H Quantification data of western blot results of $\mathrm{cCbl} / \mathrm{PKG}$ and cCbl/GAPDH. $n=3$ per group (Student's $t$ test). I TAG-23 attenuates the pro-ubiquitination effect of cCbl to PKG in $\mathrm{H} 9 \mathrm{C} 2$ cells. $n=3$ per group (one-way ANOVA analysis with Bonferroni's multiple comparison test). J TAG-23 mediated PKG degradation at the Lys48 sites in H9C2 cells. $n=3$ per group (Student's $t$ test). $* * P<0.01$, $* * * P<0.001$. Data are means \pm SD with $n=3$ independent biological replicates 
PKG ubiquitination by attenuating the interaction between PKG and cCbl. TAG-23 peptide appears to be a valuable therapeutic candidate because the crucial role of PKG and ERK in I/R injury. Our study provides evidence that peptide TAG-23 is a promising candidate for MI therapy.

To explore the mechanism underlying the effect of TAG23 peptide in reperfusion injury, we first performed a pulldown assay to identify the potential binding target of TAG23. A silver staining assay showed 2 different bands, and a total of 235 proteins that may interact with TAG-23 were identified by mass spectrometry. Among these proteins, PKG was the most highly expressed. Further coimmunoprecipitation (Co-IP) assays confirmed the interaction between TAG23 and PKG in HEK293 and H9C2 cells. However, due to the lack of a TAG-23 antibody, we failed to verify that PKG can be pulled down by TAG-23. Cyclic GMP-dependent protein kinase (PKG) belongs to the serine/threonine kinase family and is an important mediator of nitric oxide (NO) [14]. Previous studies have reported that PKGI $\alpha$ is mainly expressed in cardiomyocytes [11]. It is interesting to note that PKG, an important regulator of cardioprotection, activates ERK [7], which plays prosurvival roles, and inhibits JNK [6] and p38 kinase [11], which exert proapoptotic effects on cardiovascular diseases $[38,15]$. DOX is a frequently used chemotherapeutic agent used to treat numerous tumors [19], and it can also cause irreversible cardiac toxicity, including heart failure [40], cardiomyocyte apoptosis [17], and oxidative stress [20]. Although numerous studies have focused on DOX-induced heart failure, the underlying mechanism remains unclear [22]. In our study, we found that $\mathrm{H} / \mathrm{R}$ and DOX treatment significantly reduced the protein

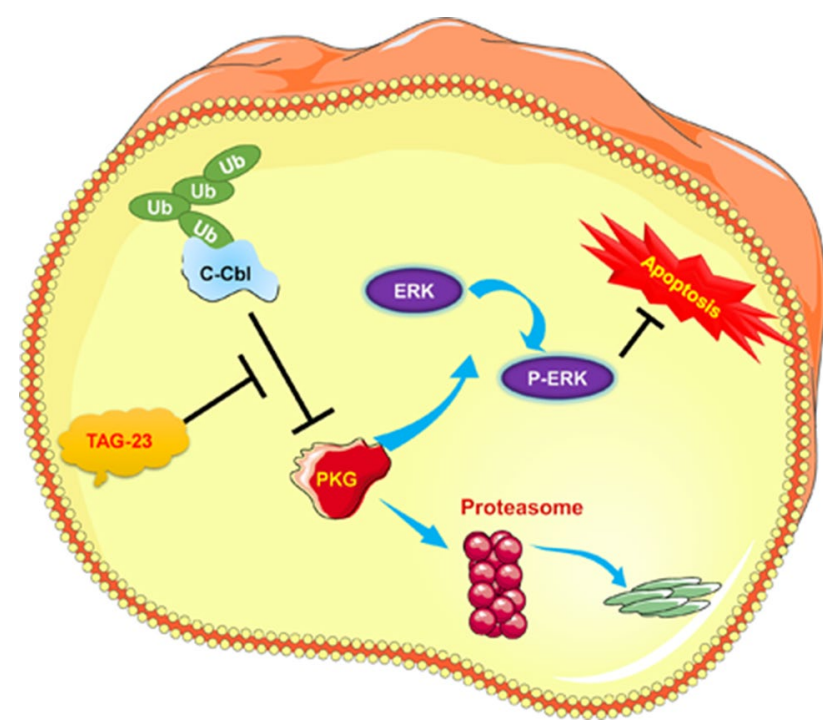

Fig. 8 Graphic abstract TAG-23 can inhibit PKG degradation by serving as a competitive binding peptide attenuate the formation of the PKG-cCbl complex level of phosphorylated ERK and that the TAG-23 peptide increased the protein level of p-ERK. However, TAG-23 had no effect on the protein level of p-p38 or p-JNK. We speculated that TAG- 23 protects cardiomyocytes against reperfusion and oxidative stress by activating the PKG-ERK signaling pathway. Thus, the downstream ERK activation is upregulated upon TAG-23 treatment.

Previous studies have shown that the half-life of PKG can be very long or rather short depending on the cell type [29]. For example, the half-life of PKG is approximately $24 \mathrm{~h}$ in vascular smooth muscle cells (VSMCs) and 60 days in platelets. However, there have been no reports regarding the specific half-life of PKG in cardiomyocytes. We found that the half-life of PKG was extended to varying degrees in cardiomyocytes and HEK293 cells. In smooth muscle cells, PKG can be degraded through the ubiquitin (Ub)/26S proteasome system (UPS), and mutation of the Ser64 autophosphorylation site to an alanine residue can block the degradation of PKG [9]. Another study showed that Ub molecules can easily bind to PKG-I under hypoxic conditions, leading to accumulation of ubiquitinated PKG-I [26]. PKG can positively regulate proteasome activities, and activation of PKG by sildenafil can reduce the accumulation of misfolded proteins by stimulating the UPS in cardiomyocytes [25, 28]. It is well established that E3 ligase is responsible for targeting specific substrate proteins for ubiquitination. Although the function and downstream genes of PKG have been extensively investigated, there have been no reports on the E3 ligase of PKG, and how $\mathrm{PKG}$ is regulated in $\mathrm{I} / \mathrm{R}$ injury remains unknown. In our study, we identified that the $\mathrm{E} 3$ ligase $\mathrm{cCbl}$ can bind with PKG and promote its degradation. Thus, we overexpressed cCbl and PKG in H9C2 and HEK293 cells to verify the influence of TAG-23 on ubiquitination. Our results demonstrated that TAG-23 attenuated the interaction between PKG and cCbl, thus reducing the degradation of PKG and alleviating cell apoptosis. However, ubiquitin possesses several different lysine residues that serve as ubiquitination sites, of which Lys48 and Lys63 are the most well characterized. As expected, mutation of the Lys48 site to an arginine residue almost completely abolished TAG-23-mediated PKG degradation, whereas there was no significant difference in PKG degradation in the presence of the K63R mutation. These data show that TAG-23 mediates PKG polyubiquitination degradation at the Lys48 site. Previous studies have also shown that autophosphorylation of Ser64 can trigger the downregulation of PKG $[9,29]$. Thus, further studies that identify the binding site between TAG-23 and PKG and determine whether Ser64 mutation can block the function of TAG-23 are warranted.

As previously reported, four healthy male volunteers were undergoing cycle exercise for $6 \mathrm{~min}$ at $77 \%$ of individual Wmax and then to exhaustion at $87-88 \%$ of Wmax. TAG-23 was induced by exercise and TAG-23 was increased 2.659 -fold 
change after exercise. Despite the endogenous peptide TAG-23 content rose about 2.659-fold after the exercise, the amount of TAG-23 induced by exercise was still limited. Nonetheless, the exogenous supplement of TAG-23 in vivo and in vitro achieved great beneficial effects. Another example is recombinant human brain natriuretic peptide. BNP level in healthy people is much lower than patients with heart failure. However, recombinant human BNP can successful applied for heart failure patients. In addition, the exercise-induced peptidome has also enable the identification of new peptides that may possess roles in different physiological processes and organ crosstalk. For example, 22 peptides derived from kininogen-1 (KNG) are significantly increased during exercise. Peptides are typically derived from the degradation of proteins, executed by the UPS system. Commonly, the protein degradation process regulated by UPS was radically and we speculated that a particular protease or peptidase might have been activated during the exercise process, which digested TAGLN and generated peptide TAG-23. Exploring the regulatory network may be an intriguing field to follow in the future. Taken together, these findings confirm that beneficial effects are mediated by peptides and other circulating factors. It is possible that several secreted peptides may exert beneficial effects, at least in part, during exercise.

Previous studies have suggested that transgelin interacts with actin stress fibers and stabilizes actin in vivo [18]. Transgelin has also been shown to be associated with a specific subpopulation of actin filament bundles that form podosomes [31]. The percentage of infarction (infarction ratio) for wild-type MI mice was measured as $11.2 \%$ while that for transgelin(-/-) mice was measured as $16.2 \%$. SAA (A traditional Chinese medicine) could stabilize the transgelin-actin complex, modulate the reorganization of the actin cytoskeleton, facilitate F-actin bundling, further enhance the contractility and blood flows of coronary arteries, and reduce infarct size. In addition to be a fundamental component of the cytoskeleton, actin has been found to carry out different functions in the cell nucleus [32]. Actin has been shown to be associated with chromatin remodeling and RNA processing [27, 32]. In our study, we found that the peptide TAG-23, which is derived from the structural protein transgelin, can alleviate cell apoptosis and MI. Structural proteins, which constitute the cell structure and allow mobility, are considered components of the cytoskeleton [30]. Previous studies have confirmed that the rapid and specific binding of Smad3 to the transgelin promoter. TGF- $\beta$ treatment resulted in rapid up-regulation of transgelin in A549 cells. Transgelin expression was specifically localized to ATII and smooth muscle cells in mouse lungs, and its expression was augmented in ATII cells in lung fibrosis. Our results may provide a certain reference value for the function of transgelin protein to reduce the area of myocardial infarction.

Our study still has some limitations. First, for further clinical application, a detailed evaluation of response time in murine models or clinical animal models is essential to fully understand the time window of cardioprotection in MI. Second, the relationship between peptide TAG- 23 and TGF- $\beta$ in cardiomyocytes remain elusive. Exploring the regulatory work in cardiomyocytes will further explain the effect of TAG- 23 .

In summary, we performed a comprehensive functional analysis of the peptide TAG-23, which can inhibit cell apoptosis and oxidative stress in vitro and ameliorate MI and heart failure in vivo. We also showed that targeting the PKG-cCbl interaction with an endogenous pharmacological peptide directly reduced the degradation of PKG, thus allowing activation of its downstream protein ERK. Our study provides a new approach for treating MI.

Supplementary Information The online version contains supplementary material available at https://doi.org/10.1007/s00395-021-00878-4.

Author contributions $\mathrm{ZC}$ and $\mathrm{HZ}$ designed and performed the experiments, analyzed data, and wrote the manuscript. XW and LZ performed some in vitro experiments. QZ performed the mass spectrometry analysis and analyzed data. MF and DH participated in the animal experiments. HL performed the bioinformation analysis. LQ (corresponding author) designed, supervised the study, and performed manuscript editing.

Funding This work was supported by grants from the National Natural Science Foundation of China (NO.81570209, 81873540, 81971407), Postgraduate Research \& Practice Innovation Program of Jiangsu Province (NO. KYCX19_1153), Key Clinical Frontier Technology Project of Department of Science and Technology of Jiangsu Provincial (NO. BE2019752), 333 High Level Talent Project of Jiangsu Province (NO. LGY2018056), Natural Science Foundation of Zhejiang Province (NO.LY20H020001).

Data availability Original data of RNA-sequencing and mass-spec submitted with the manuscript. Data associated with temporal quantification of the exercise-regulated peptidome are available in project accession PXD004781 (username: reviewer61660@ebi.ac.uk; password: NaHrEPqj).

\section{Declarations}

Conflict of interest The authors declare that they have no conflict of interest.

Ethics approval All animal studies were approved by the Animal Care and Ethics Committee of Nanjing Medical University.

Consent to participate Not applicable.

Consent for publication Not applicable.

Open Access This article is licensed under a Creative Commons Attribution 4.0 International License, which permits use, sharing, adaptation, distribution and reproduction in any medium or format, as long as you give appropriate credit to the original author(s) and the source, provide a link to the Creative Commons licence, and indicate if changes were made. The images or other third party material in this article are included in the article's Creative Commons licence, unless indicated otherwise in a credit line to the material. If material is not included in 
the article's Creative Commons licence and your intended use is not permitted by statutory regulation or exceeds the permitted use, you will need to obtain permission directly from the copyright holder. To view a copy of this licence, visit http://creativecommons.org/licenses/by/4.0/.

\section{References}

1. Aihara K, Osaka M, Yoshida M (2014) Oral administration of the milk casein-derived tripeptide Val-Pro-Pro attenuates highfat diet-induced adipose tissue inflammation in mice. Br J Nutr 112:513-519. https://doi.org/10.1017/S0007114514001147

2. Assinder SJ, Stanton JA, Prasad PD (2009) Transgelin: an actinbinding protein and tumour suppressor. Int J Biochem Cell Biol 41:482-486. https://doi.org/10.1016/j.biocel.2008.02.011

3. Bei Y, Pan LL, Zhou Q, Zhao C, Xie Y, Wu C, Meng X, Gu H, Xu J, Zhou L, Sluijter JPG, Das S, Agerberth B, Sun J, Xiao J (2019) Cathelicidin-related antimicrobial peptide protects against myocardial ischemia/reperfusion injury. BMC Med 17:42. https:// doi.org/10.1186/s12916-019-1268-y

4. Cahill TJ, Choudhury RP, Riley PR (2017) Heart regeneration and repair after myocardial infarction: translational opportunities for novel therapeutics. Nat Rev Drug Discov 16:699-717. https://doi. org/10.1038/nrd.2017.106

5. Chan SH, Sun EY, Chang AY (2010) Extracellular signalregulated kinase $1 / 2$ plays a pro-life role in experimental brain stem death via MAPK signal-interacting kinase at rostral ventrolateral medulla. J Biomed Sci 17:17. https://doi.org/10.1186/ 1423-0127-17-17

6. Das A, Smolenski A, Lohmann SM, Kukreja RC (2006) Cyclic GMP-dependent protein kinase Ialpha attenuates necrosis and apoptosis following ischemia/reoxygenation in adult cardiomyocyte. J Biol Chem 281:38644-38652. https://doi.org/10.1074/jbc. M606142200

7. Das A, Salloum FN, Xi L, Rao YJ, Kukreja RC (2009) ERK phosphorylation mediates sildenafil-induced myocardial protection against ischemia-reperfusion injury in mice. Am J Physiol Heart Circ Physiol 296:H1236-1243. https://doi.org/10.1152/ ajpheart.00100.2009

8. Del Re DP, Amgalan D, Linkermann A, Liu Q, Kitsis RN (2019) Fundamental mechanisms of regulated cell death and implications for heart disease. Physiol Rev 99:1765-1817. https://doi. org/10.1152/physrev.00022.2018

9. Dey NB, Busch JL, Francis SH, Corbin JD, Lincoln TM (2009) Cyclic GMP specifically suppresses Type-Ialpha cGMP-dependent protein kinase expression by ubiquitination. Cell Signal 21:859-866. https://doi.org/10.1016/j.cellsig.2009.01.014

10. Ding X, Jia X, Wang C, Xu J, Gao SJ, Lu C (2018) A DHX9lncRNA-MDM2 interaction regulates cell invasion and angiogenesis of cervical cancer. Cell Death Differ 9:1750-1765. https://doi.org/10.1038/s41418-018-0242-0

11. Fiedler B, Feil R, Hofmann F, Willenbockel C, Drexler H, Smolenski A, Lohmann SM, Wollert KC (2006) cGMP-dependent protein kinase type I inhibits TAB1-p38 mitogen-activated protein kinase apoptosis signaling in cardiac myocytes. J Biol Chem 281:32831-32840. https://doi.org/10.1074/jbc.M6034 16200

12. Fiuza-Luces C, Garatachea N, Berger NA, Lucia A (2013) Exercise is the real polypill. Physiology (Bethesda) 28:330-358. https://doi.org/10.1152/physiol.00019.2013

13. Frankenreiter S, Bednarczyk P, Kniess A, Bork NI, Straubinger J, Koprowski P, Wrzosek A, Mohr E, Logan A, Murphy MP, Gawaz M, Krieg T, Szewczyk A, Nikolaev VO, Ruth P, Lukowski R (2017) cGMP-Elevating compounds and ischemic conditioning provide cardioprotection against ischemia and reperfusion injury via cardiomyocyte-specific BK channels. Circulation 136:2337-2355. https://doi.org/10.1161/circulatio naha.117.028723

14. Heusch G (2017) Cardioprotection is alive but remains enigmatic: the nitric oxide-protein kinases-mitochondria signaling axis. Circulation 136:2356-2358. https://doi.org/10.1161/circulationaha. 117.031978

15. Heusch G (2020) Myocardial ischaemia-reperfusion injury and cardioprotection in perspective. Nat Rev Cardiol 17:773-789. https://doi.org/10.1038/s41569-020-0403-y

16. Gallo S, Gatti S, Sala V, Albano R, Costelli P, Casanova E, Comoglio PM, Crepaldi T (2014) Agonist antibodies activating the Met receptor protect cardiomyoblasts from cobalt chlorideinduced apoptosis and autophagy. Cell Death Dis 5:e1185. https:// doi.org/10.1038/cddis.2014.155

17. Hawley JA, Hargreaves M, Joyner MJ, Zierath JR (2014) Integrative biology of exercise. Cell 159:738-749. https://doi.org/10. 1016/j.cell.2014.10.029

18. Hou Z, Qin X, Hu Y, Zhang X, Li G, Wu J, Li J, Sha J, Chen J, Xia J, Wang L, Gao F (2019) Longterm exercise-derived exosomal miR-342-5p. Circ Res 124:1386-1400. https://doi.org/10.1161/ CIRCRESAHA.118.314635

19. Kumar SN, Konorev EA, Aggarwal D, Kalyanaraman B (2011) Analysis of proteome changes in doxorubicin-treated adult rat cardiomyocyte. J Proteomics 74:683-697. https://doi.org/10.1016/j. jprot.2011.02.013

20. Lawson D, Harrison M, Shapland C (1997) Fibroblast transgelin and smooth muscle SM22alpha are the same protein, the expression of which is down-regulated in many cell lines. Cell Motil Cytoskeleton 38:250-257. https://doi.org/10.1002/(SICI)10970169(1997)38:3\%3c250::AID-CM3\%3e3.0.CO;2-9

21. Li M, Sala V, De Santis MC, Cimino J, Cappello P, Pianca N, Di Bona A, Margaria JP, Martini M, Lazzarini E, Pirozzi F, Rossi L, Franco I, Bornbaum J, Heger J, Rohrbach S, Perino A, Tocchetti CG, Lima BHF, Teixeira MM, Porporato PE, Schulz R, Angelini A, Sandri M, Ameri P, Sciarretta S, Lima-Júnior RCP, Mongillo M, Zaglia T, Morello F, Novelli F, Hirsch E, Ghigo A (2018) Phosphoinositide 3-kinase gamma inhibition protects from anthracycline cardiotoxicity and reduces tumor growth. Circulation 138:696-711. https://doi.org/10.1161/CIRCULATIONAHA. 117.030352

22. Liu D, Ma Z, Di S, Yang Y, Yang J, Xu L, Reiter RJ, Qiao S, Yuan J (2018) AMPK/PGC1 $\alpha$ activation by melatonin attenuates acute doxorubicin cardiotoxicity via alleviating mitochondrial oxidative damage and apoptosis. Free Radic Biol Med 129:59-72. https:// doi.org/10.1016/j.freeradbiomed.2018.08.032

23. McCormick LM, Hoole SP, White PA, Read PA, Axell RG, Clarke SJ, O'Sullivan M, West NEJ, Dutka DP (2015) Pre-treatment with glucagon-like Peptide-1 protects against ischemic left ventricular dysfunction and stunning without a detected difference in myocardial substrate utilization. JACC Cardiovasc Interv 8:292-301. https://doi.org/10.1016/j.jcin.2014.09.014

24. Minotti G, Menna P, Salvatorelli E, Cairo G, Gianni L (2004) Anthracyclines: molecular advances and pharmacologic developments in antitumor activity and cardiotoxicity. Pharmacol Rev 56:185-229. https://doi.org/10.1124/pr.56.2.6

25. Parker BL, Burchfield JG, Clayton D, Geddes TA, Payne RJ, Kiens B, Wojtaszewski JFP, Richter EA, James DE (2017) Multiplexed temporal quantification of the exercise-regulated plasma peptidome. Mol Cell Proteomics 16:2055-2068. https://doi.org/ 10.1074/mcp.RA117.000020

26. Petzelbauer P, Zacharowski PA, Miyazaki Y, Friedl P, Wickenhauser G, Castellino FJ, Gröger M, Wolff K, Zacharowski $\mathrm{K}$ (2005) The fibrin-derived peptide Bbeta15-42 protects the 
myocardium against ischemia-reperfusion injury. Nat Med 11:298-304. https://doi.org/10.1038/nm1198

27. Rainer PP, Kass DA (2016) Old dog, new tricks: novel cardiac targets and stress regulation by protein kinase G. Cardiovasc Res 111:154-162. https://doi.org/10.1093/cvr/cvw107

28. Ramchandran R, Pilipenko E, Bach L, Raghavan A, Reddy SP, Raj JU (2012) Hypoxic regulation of pulmonary vascular smooth muscle cyclic guanosine monophosphate-dependent kinase by the ubiquitin conjugating system. Am J Respir Cell Mol Biol 46:323330. https://doi.org/10.1165/rcmb.2011-0165OC

29. Rando OJ, Zhao K, Crabtree GR (2000) Searching for a function for nuclear actin. Trends Cell Biol 10:92-97. https://doi.org/10. 1016/s0962-8924(99)01713-4

30. Ranek MJ, Terpstra EJ, Li J, Kass DA, Wang X (2013) Protein kinase g positively regulates proteasome-mediated degradation of misfolded proteins. Circulation 128:365-376. https://doi.org/10. 1161/CIRCULATIONAHA.113.001971

31. Sellak H, Choi CS, Dey NB, Lincoln TM (2013) Transcriptional and post-transcriptional regulation of cGMP-dependent protein kinase (PKG-I): pathophysiological significance. Cardiovasc Res 97:200-207. https://doi.org/10.1093/cvr/cvs327

32. Shakhov AS, Dugina VB, Alieva IB (2019) Structural features of actin cytoskeleton required for endotheliocyte barrier function. Biochemistry (Mosc) 84:358-369. https://doi.org/10.1134/S0006 297919040035

33. Uribe R, Jay D (2009) A review of actin binding proteins: new perspectives. Mol Biol Rep 36:121-125. https://doi.org/10.1007/ s11033-007-9159-2

34. Vanhaverbeke M, Bogaerts K, Sinnaeve PR, Janssens L, Armstrong PW, Van de Werf F (2019) Prevention of cardiogenic shock after acute myocardial infarction. Circulation 139:137-139. https://doi.org/10.1161/CIRCULATIONAHA.118.036536
35. Vega RB, Konhilas JP, Kelly DP, Leinwand LA (2017) Molecular mechanisms underlying cardiac adaptation to exercise. Cell Metab 25:1012-1026. https://doi.org/10.1016/j.cmet.2017.04.025

36. Wang ZV, Deng Y, Gao N, Pedrozo Z, Li DL, Morales CR, Criollo A, Luo X, Tan W, Jiang N, Lehrman MA, Rothermel BA, Lee AH, Lavandero S, Mammen PPA, Ferdous A, Gillette TG, Scherer PE, Hill JA (2014) Spliced X-box binding protein 1 couples the unfolded protein response to hexosamine biosynthetic pathway. Cell 156:1179-1192. https://doi.org/10.1016/j.cell.2014.01.014

37. Yu LM, Di WC, Dong X, Li Z, Zhang Y, Xue XD, Xu YL, Zhang J, Xiao X, Han JS, Liu Y, Yang Y, Wang HS (2018) Melatonin protects diabetic heart against ischemia-reperfusion injury, role of membrane receptor-dependent cGMP-PKG activation. Biochim Biophys Acta Mol Basis Dis 1864:563-578. https://doi.org/10. 1016/j.bbadis.2017.11.023

38. Yu LM, Yang G, Zhang XJ, Wang P, Weng XY, Yang YY, Li ZL, Fang MM, Xu Y, Sun AJ, Ge JB (2018) Megakaryocytic Leukemia 1 (MKL1) bridges epigenetic activation of NADPH oxidase in macrophages to cardiac ischemia-reperfusion injury. Circulation 138(24):2820-2836. https://doi.org/10.1161/circulationaha.118. 035377

39. Zhang T, Zhang Y, Cui M, Jin L, Wang Y, Lv F, Liu Y, Zheng W, Shang H, Zhang J, Zhang M, Wu H, Guo J, Zhang X, Hu X, Cao CM, Xiao RP (2016) CaMKII is a RIP3 substrate mediating ischemia- and oxidative stress-induced myocardial necroptosis. Nat Med 22:175-182. https://doi.org/10.1038/nm.4017

40. Zhang ZW, Yang ZM, Zheng YC, Chen ZD (2010) Transgelin induces apoptosis of human prostate $\mathrm{LNCaP}$ cells through its interaction with p53. Asian J Androl 12:186-195. https://doi.org/ 10.1038/aja.2009.76 